\title{
Dynamics on K3 Surfaces: Salem Numbers and Siegel Disks
}

\section{Citation}

McMullen, Curtis T. 2002. Dynamics on K3 surfaces: Salem numbers and Siegel disks. Journal fur die Reine und Angewandte Mathematik 2002(545): 201-233. Revised 2005.

\section{Published Version}

doi:10.1515/crll.2002.036

\section{Permanent link}

http://nrs.harvard.edu/urn-3:HUL.InstRepos:3446014

\section{Terms of Use}

This article was downloaded from Harvard University's DASH repository, and is made available under the terms and conditions applicable to Other Posted Material, as set forth at http:// nrs.harvard.edu/urn-3:HUL.InstRepos:dash.current.terms-of-use\#LAA

\section{Share Your Story}

The Harvard community has made this article openly available.

Please share how this access benefits you. Submit a story.

Accessibility 


\title{
Dynamics on K3 surfaces: Salem numbers and Siegel disks
}

\author{
Curtis T. McMullen
}

19 January, 2001

\begin{abstract}
This paper presents the first examples of K3 surface automorphisms $f: X \rightarrow X$ with Siegel disks (domains on which $f$ acts by an irrational rotation). The set of such examples is countable, and the surface $X$ must be non-projective to carry a Siegel disk.

These automorphisms are synthesized from Salem numbers of degree 22 and trace -1 , which play the role of the leading eigenvalue for $f^{*} \mid H^{2}(X)$. The construction uses the Torelli theorem, the Atiyah-Bott fixed-point theorem and results from transcendence theory.
\end{abstract}

\section{Contents}

$1 \quad$ Introduction . . . . . . . . . . . . . . . . . . 1

2 K3 surfaces . . . . . . . . . . . . . . 7

3 Automorphisms of K3 surfaces . . . . . . . . . . . . 11

4 Ergodic dynamics on Kummer surfaces . . . . . . . . . . . . . 14

5 Siegel disks and transcendence theory . . . . . . . . . 18

6 Holomorphic Lefschetz numbers . . . . . . . . . . . . . . . 20

7 Siegel disks on K3 surfaces . . . . . . . . . . . . . . . . 22

8 Lattices in number fields . . . . . . . . . . . . . . . . 24

9 From Salem numbers to automorphisms . . . . . . . . . . . 29

10 Examples of Siegel disks . . . . . . . . . . . . . . . 30

11 Limits of Kähler-Einstein metrics . . . . . . . . . . . . . . 34

Research supported in part by the NSF.

2000 Mathematics Subject Classification: 37F50 (11R06, 14J50, 32H50). 


\section{Introduction}

The first dynamically interesting automorphisms of compact complex manifolds arise on K3 surfaces.

Indeed, automorphisms of curves are linear (genus 0 or 1) or of finite order (genus 2 or more). Similarly, automorphisms of most surfaces (including $\mathbb{P}^{2}$, surfaces of general type and ruled surfaces) are either linear, finite order or skew-products over automorphisms of curves. Only K3 surfaces, Enriques surfaces, complex tori and certain non-minimal rational surfaces admit automorphisms of positive topological entropy [Ca2]. The automorphisms of tori are linear, and the Enriques examples are double-covered by K3 examples.

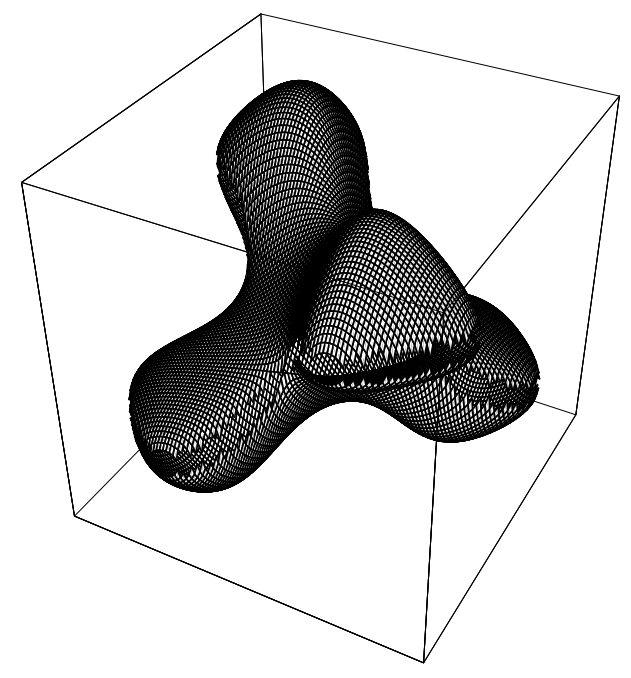

Figure 1 . A K3 surface in $\mathbb{P}^{1} \times \mathbb{P}^{1} \times \mathbb{P}^{1}$.

Over $\mathbb{R}$. To give an idea of the richness of dynamics on K3 surfaces, consider a smooth hypersurface

$$
X \subset \mathbb{P}^{1} \times \mathbb{P}^{1} \times \mathbb{P}^{1}
$$

of degree $(2,2,2)$, defined by the affine equation

$$
\left(1+x^{2}\right)\left(1+y^{2}\right)\left(1+z^{2}\right)+A x y z=2, \quad A \in \mathbb{R} .
$$

By the adjunction formula, the canonical bundle of $X$ is trivial, and by the 

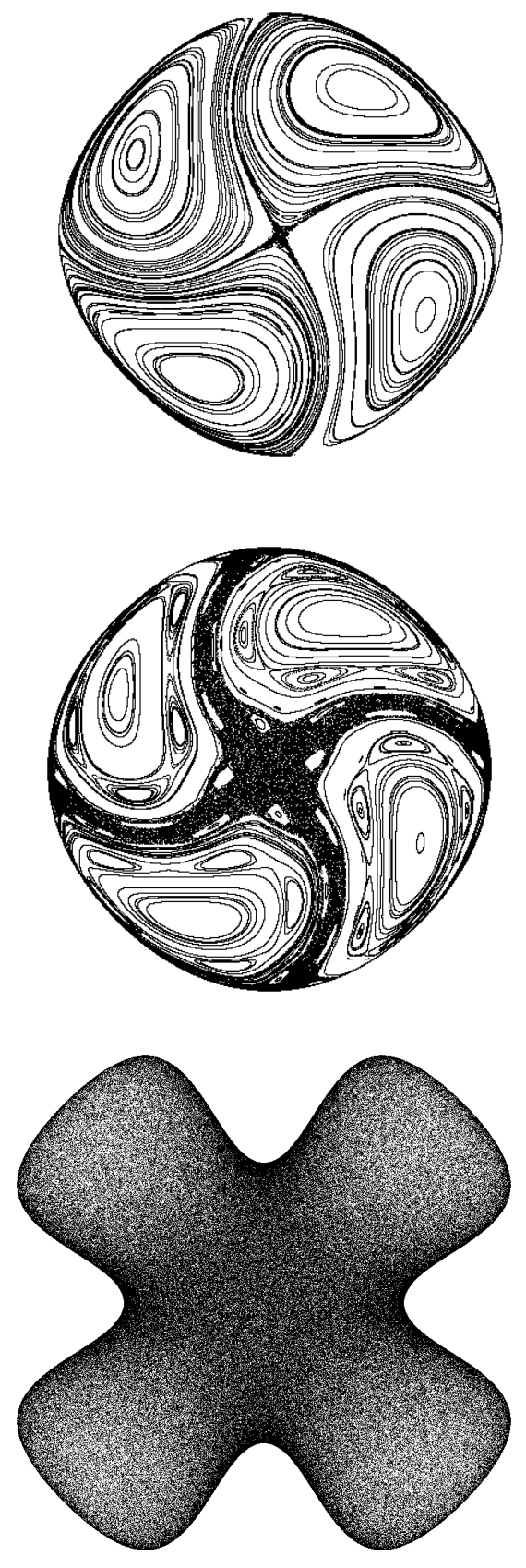

Figure 2. Dynamics on real K3 surfaces. 
Lefschetz hyperplane theorem $b_{1}(X)=0$, so $X$ is a K3 surface. Its real points $X(\mathbb{R})$ for $A=8$ are shown in Figure 1 .

A line through $p \in X$ parallel to the $x$-axis passes through a unique second point $\iota_{x}(p) \in X$, and similarly for $y$ and $z$. For our dynamical system on $X$, we take the composition of these 3 involutions,

$$
f=\iota_{x} \circ \iota_{y} \circ \iota_{z}
$$

The automorphism $f \mid X(\mathbb{R})$ is area-preserving; indeed, any K3 surface carries a nowhere-zero holomorphic $(2,0)$-form $\eta$, which in turn determines an invariant measure $|\eta|$ on $X(\mathbb{R})$.

Orbits of $f: X(\mathbb{R}) \rightarrow X(\mathbb{R})$, as viewed from above, are shown in Figure 2 for $A=2,2.5$ and 8 . When $A=2$ (top) the dynamics is dominated by the elliptic islands predicted by KAM theory; there are many invariant disks and no dense orbits. For $A=8$ the dynamics on $X(\mathbb{R})$ seems to be ergodic. The intermediate parameter $A=2.5$ exhibits a mixture of behaviors: elliptic islands seem to coexist with an ergodic component of positive measure.

The dynamics in these real examples is typical for area-preserving maps on surfaces. Aside from the existence of elliptic islands, little is rigorously known about the ergodic theory of such mappings; cf. [Mak1], [Mak2].

Over $\mathbb{C}$. In this paper we will be concerned with the dynamics of automorphisms of complex K3 surfaces, $f: X \rightarrow X$.

Every automorphism preserves the measure $\eta \wedge \bar{\eta}$ on $X$ determined by a nowhere zero holomorphic $(2,0)$-form $\eta$. Using Kummer surfaces, it is easy to construct ergodic K3 surface automorphisms from complex tori (§4). It is natural to ask if all automorphisms of positive topological entropy are ergodic, or at least have a dense orbit.

Siegel disks. Let us say a linear map $F\left(z_{1}, z_{2}\right)=\left(\lambda_{1} z_{1}, \lambda_{2} z_{2}\right)$ is an irrational rotation if $\left|\lambda_{1}\right|=\left|\lambda_{2}\right|=1$ and $F$ has dense orbits on $S^{1} \times S^{1}$. A domain $U \subset X$ is a Siegel disk for $f$ if $f(U)=U$ and $f \mid U$ is analytically conjugate to $F \mid \Delta^{2}$ for some irrational rotation $F$. (Here $\Delta=\{z:|z|<1\}$.)

Like an elliptic island on $X(\mathbb{R})$, a Siegel disk on $X$ is an obstruction to ergodicity and to the existence of dense orbits. The main result of this paper, achieved in $\S 10$, is:

Theorem 1.1 There exist K3 surface automorphisms with Siegel disks.

Every such automorphism has positive topological entropy.

Unfortunately, these Siegel disks are invisible to us: they live on nonprojective K3 surfaces, and we can only detect them implicitly, through Hodge theory and dynamics on the cohomology. Indeed, in $\S 7$ we show: 
Theorem 1.2 There are at most countably many K3 surface automorphisms with Siegel disks, up to isomorphism; and there are no Siegel disks on projective K3 surfaces.

Application: Kähler-Einstein metrics. Recall that any class in the Kähler cone $C_{X} \subset H^{1,1}(X)_{\mathbb{R}}$ of a K3 surface is represented by the symplectic form $\omega$ of a unique Kähler-Einstein metric. It would be interesting to understand the behavior of these metrics as $[\omega]$ tends to $\partial C_{X}$. In $\S 11$ we use dynamics to show that $[\omega]$ can collapse on an open set.

Theorem 1.3 Let $f: X \rightarrow X$ be a K3 surface automorphism with a Siegel disk $U$. Then there exists a sequence of Kähler-Einstein symplectic forms such that

$$
\left[\omega_{n}\right] \rightarrow \xi \in \partial C_{X}, \quad \xi \neq 0,
$$

while $\omega_{n} \mid U \rightarrow 0$ uniformly on compact sets.

Action on cohomology. We now turn to a more detailed account of the dynamics of automorphisms.

For any $\mathrm{K} 3$ surface, the space $H^{2}(X, \mathbb{Z})$ is an even, unimodular lattice of signature $(3,19)$ with respect to the intersection form. By the Torelli theorem for K3 surfaces, Aut $(X)$ acts faithfully on $H^{2}(X)$. Our approach to dynamics on $X$ is via the action on $H^{2}(X)$.

Two useful invariants, discussed in $\S 3$, are:

$\lambda(f)$ - the spectral radius of $f^{*} \mid H^{2}(X)$; and

$\delta(f)$ - the eigenvalue of $f^{*}$ on the line $H^{2,0}(X)=\mathbb{C} \cdot \eta$.

The topological entropy of $f$ is given by $h(f)=\log \lambda(f) \geq 0$. When $f$ has positive entropy, $\lambda(f)>1$ is the unique eigenvalue of $f^{*} \mid H^{2}(X)$ outside the unit circle. Thus $\lambda(f)$ is a Salem number: an algebraic unit whose conjugates other than $\lambda(f)^{ \pm 1}$ lie on the unit circle.

The map $f$ preserves $\int_{X} \eta \wedge \bar{\eta}$, and so $|\delta(f)|=1$. We refer to $\delta(f)$ as the determinant of $f$, since

$$
\operatorname{det} D f_{p}=\delta(f)
$$

at any fixed-point $p$ of $X$. When $X$ is projective, $\delta(f)$ is a root of unity, and thus $p$ cannot be the center of a Siegel disk. This is why we must look to non-projective surfaces for Siegel disk examples. ${ }^{1}$

Construction of automorphisms. Here is a sketch of the construction of automorphisms with Siegel disks.

\footnotetext{
'Resonant' rotation domains, where $\operatorname{det} D f_{p}$ is a root of unity, seem unlikely to exist $(\S 7)$, but we do not know how to rule them out.
} 
1. Let $\lambda>1$ be a degree 22 Salem number with minimal polynomial $S(x) \in \mathbb{Z}[x]$. Our first goal is to construct a K3 surface automorphism $f: X \rightarrow X$ such that $\lambda(f)=\lambda$ and $S(x)$ is the characteristic polynomial of $f^{*} \mid H^{2}(X)$.

2. Let $B=\mathbb{Z}[y] /(S(y))$, and let $K$ be the field of fractions of $B$. Let $U(x)$ be a unit in the subring of $B$ generated by $x=y+y^{-1}$. We make $B$ into a lattice by defining the inner product

$$
\left\langle g_{1}, g_{2}\right\rangle_{B(U)}=\operatorname{Tr}_{\mathbb{Q}}^{K}\left(\frac{U(x) g_{1}(y) g_{2}\left(y^{-1}\right)}{R^{\prime}(x)}\right),
$$

where $R(x)$ is the minimal polynomial of $\lambda+\lambda^{-1}$. Assuming $|S( \pm 1)|=$ 1 (that is, $\lambda$ is unramified) and $U$ is suitably chosen, the above inner product makes $B(U)$ into an even, unimodular lattice of signature $(3,19)$. See $\S 8$.

3. Let $F: B \rightarrow B$ be multiplication by $y$. Then $F$ is an isometry of the lattice $B(U)$. There is a unique eigenspace $E \subset B(U) \otimes \mathbb{C}$ of $F+F^{-1}$ with signature $(2,0)$. Writing

$$
E=H^{2,0} \oplus H^{0,2}
$$

as a sum of eigenspaces of $F$ with eigenvalues $\delta$ and $\delta^{-1}$ respectively, we obtain a Hodge structure on $B(U)$ invariant under $F$.

4. By surjectivity of the period mapping, there is a K3 surface $X$ and an isomorphism of lattices $B(U) \cong H^{2}(X, \mathbb{Z})$ respecting the Hodge structure. The subspace $H^{1,1}(X)$ is sufficiently irrational that $\operatorname{Pic}(X)=0$; in particular, $X$ is nonprojective.

5. By the Torelli theorem, there is an automorphism $f: X \rightarrow X$ making the diagram

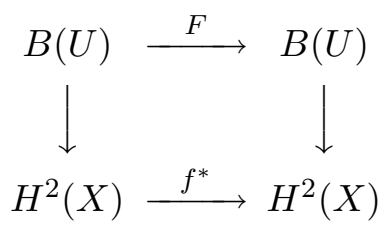

commutative (§9). Thus $\lambda(f)=\lambda$ and $\delta(f)=\delta$. The fixed-points of $f$ are isolated because the only subvarieties of $X$ are points. 
6. Now suppose the trace of $\lambda$ is -1 . Then $f$ has a unique fixed-point $p \in X$, since its Lefschetz number is given by

$$
L(f)=\operatorname{Tr} f^{*} \mid\left(H^{0} \oplus H^{2} \oplus H^{4}\right)=1-1+1=1 .
$$

The Atiyah-Bott formula relates $\operatorname{Tr} D f_{p}$ to $\operatorname{Tr} f^{*} \mid H^{2,0}(X)=\delta$, and we already know that $\operatorname{det} D f_{p}=\delta$. Thus the eigenvalues $\alpha, \beta$ of $D f_{p}$ are determined by $\delta$. See $\S 6$.

7. For suitable values of $\delta, D f_{p}$ is an irrational rotation. That is, its eigenvalues $\alpha, \beta$ lie on $S^{1}$ and are multiplicatively independent, meaning

$$
\alpha^{i}=\beta^{j} \Longleftrightarrow(i, j)=(0,0) .
$$

The eigenvalues lie on $S^{1}$ if $\tau=\delta+\delta^{-1}>1-2 \sqrt{2}$, and they are multiplicatively independent if $\tau$ has a conjugate $\tau^{\prime}<1-2 \sqrt{2}$. See $\S 7$.

8. Assume now that the algebraic numbers $\alpha$ and $\beta$ are multiplicatively independent. Then they are jointly Diophantine, by a result of Fel'dman. That is, there exist $C, M>0$ such that

$$
\left|\alpha^{i} \beta^{j}-1\right|>C(|i|+|j|)^{-M}
$$

for all $(i, j) \neq(0,0)$. The proof uses transcendence theory and the Gel'fond-Baker method.

9. By a result of Siegel and Sternberg, once the eigenvalues of $D f_{p}$ are jointly Diophantine, $f$ is locally linearizable. We conclude that $f$ has a Siegel disk centered at $p$. See $\S 5$.

10. To complete the construction, we must exhibit unramified degree 22 Salem polynomials $S(x)$ of trace -1 , and units $U(x)$, such that the root $\delta$ of $S(x)$ satisfies the bounds required in step 7 . We note that Salem numbers with trace -1 are rather rare; there are only finitely many such numbers of degree 22, and there are no known Salem numbers of trace $<-1$.

The desired polynomials, located by an intensive computer search, are presented in $\S 10$.

Questions. In conclusion we remark that many basic questions concerning dynamics on projective K3 surfaces remain open. For example, let $f: X \rightarrow$ 
$X$ be an automorphism of a projective K3 surface with positive entropy. Can $f$ have a rotation domain? Does $f$ have a dense orbit? Is $f$ ergodic?

Notes and references. For more about dynamics on complex surfaces, see the work of Cantat [Ca2], [Ca1]. Dynamical systems on certain cubic surfaces are considered by Fried in [Fr]; see also [Br].

For an arithmetic perspective on K3 surface dynamics, see work of Silverman, Mazur and Wang: [Sil], [Maz], [Wa]. Silverman studies the intersection of $(1,1)$ and $(2,2)$ hypersurfaces in $\mathbb{P}^{2} \times \mathbb{P}^{2}$, while Wang studies $(2,2,2)$ surfaces in $\mathbb{P}^{1} \times \mathbb{P}^{1} \times \mathbb{P}^{1}$ as we do above.

Acknowledgements. I would like to thank B. Mazur and B. Gross for many stimulating discussions. Mazur introduced me to dynamics on K3 surfaces in 1992, including the examples of $(2,2,2)$ hypersurfaces presented above; this paper grew out of our dialog. Gross helped engineer the numbertheoretic construction of unimodular lattices; these matters are developed in more detail in $[\mathrm{GM}]$. I would also like to thank D. Allcock, W. Stein and the referees.

\section{$2 \quad$ K3 surfaces}

This section provides a resumé of basic facts about K3 surfaces and maps between them; for details see [BPV], [Bv2]. We start with some terminology concerning lattices, to streamline the discussion of the intersection form.

Lattices. A lattice is a finitely-generated free abelian group $L \cong \mathbb{Z}^{n}$ equipped with a non-degenerate symmetric bilinear form (or inner product) $\langle x, y\rangle_{L} \in \mathbb{Z}$. The lattice $L$ is even if $\langle x, x\rangle_{L} \in 2 \mathbb{Z}$ for all $x \in L$; otherwise $L$ is odd. If the inner product gives an isomorphism between $L$ and $L^{*}=\operatorname{Hom}(L, \mathbb{Z})$, then $L$ is unimodular. We say $L$ has signature $(p, q)$ if the quadratic form $\langle x, x\rangle_{L}$ on $L \otimes \mathbb{R} \cong \mathbb{R}^{n}$ is equivalent to

$$
x_{1}^{2}+\cdots+x_{p}^{2}-x_{p+1}^{2}-\cdots-x_{p+q}^{2} .
$$

If $(p, q)=(n, 0)$ or $(0, n)$, then $L$ is definite; otherwise $L$ is an indefinite lattice.

Any two even, indefinite, unimodular lattices with the same signature are isomorphic. There exists an even unimodular lattice with signature $(p, q)$ iff $p \equiv q \bmod 8$. See [Ser1, §5], [MH].

K3 surfaces. A complex surface $X$ is a connected compact complex manifold with $\operatorname{dim}_{\mathbb{C}} X=2$. If $X$ is simply-connected and the canonical bundle of $X$ is trivial, then $X$ is a K3 surface. All K3 surfaces are diffeomorphic. 
Intersection form. Let $X$ be a K3 surface, and let

$$
(C, D) \mapsto C \cdot D \in \mathbb{Z}
$$

denote the intersection form on the middle-dimensional cohomology group $H^{2}(X, \mathbb{Z})$. This form makes $H^{2}(X, \mathbb{Z})$ into an even unimodular lattice of signature $(3,19)$. As remarked above, all such lattices are isomorphic. Thus in an appropriate basis, the intersection form is given by $C \cdot D=C Q D^{t}$ where $Q=3 H \oplus 2\left(-E_{8}\right), H=\left(\begin{array}{ll}0 & 1 \\ 1 & 0\end{array}\right)$ and

$$
E_{8}=\left(\begin{array}{cccccccc}
2 & 0 & 0 & -1 & 0 & 0 & 0 & 0 \\
0 & 2 & -1 & 0 & 0 & 0 & 0 & 0 \\
0 & -1 & 2 & -1 & 0 & 0 & 0 & 0 \\
-1 & 0 & -1 & 2 & -1 & 0 & 0 & 0 \\
0 & 0 & 0 & -1 & 2 & -1 & 0 & 0 \\
0 & 0 & 0 & 0 & -1 & 2 & -1 & 0 \\
0 & 0 & 0 & 0 & 0 & -1 & 2 & -1 \\
0 & 0 & 0 & 0 & 0 & 0 & -1 & 2
\end{array}\right)
$$

defines the positive-definite even unimodular form of rank 8. For brevity we write $C^{2}=C \cdot C$.

Since $X$ is simply-connected, its other integral cohomology groups are given by $H^{0}(X, \mathbb{Z})=H^{4}(X, \mathbb{Z})=\mathbb{Z}$ and $H^{1}(X, \mathbb{Z})=H^{3}(X, \mathbb{Z})=0$.

Signatures. The intersection form determines a Hermitian inner product on $H^{2}(X, \mathbb{C})=H^{2}(X, \mathbb{Z}) \otimes \mathbb{C}$. We say a subspace $S \subset H^{2}(X, \mathbb{C})$ has signature $(p, q)$ if $S=\bar{S}$ and the intersection pairing on $S$ is non-degenerate, with signature $(p, q)$. In this case we have $H^{2}(X, \mathbb{C})=S \oplus S^{\perp}$, and $S^{\perp}$ has signature $(3-p, 19-q)$.

Hodge structure. The complex structure of $X$ is reflected in the Hodge decomposition

$$
H^{2}(X, \mathbb{C})=H^{2,0}(X) \oplus H^{1,1}(X) \oplus H^{0,2}(X) .
$$

Here $H^{1,1}=\overline{H^{1,1}}$ and $H^{2,0}=\overline{H^{0,2}}$. Since the canonical bundle of $X$ is trivial, we have

$$
H^{2,0}(X)=\mathbb{C} \cdot \eta
$$

where $\eta$ is a nowhere vanishing holomorphic $(2,0)$-form, locally given by $\eta=\eta\left(z_{1}, z_{2}\right) d z_{1} \wedge d z_{2}$. Noting that

$$
\eta \cdot \bar{\eta}=\int_{X} \eta \wedge \bar{\eta}=4 \int_{X}|\eta|^{2}>0
$$

we find the orthogonal subspaces $H^{1,1}$ and $H^{2,0} \oplus H^{0,2}$ have signatures $(1,19)$ and $(2,0)$ respectively. 
Projective surfaces. The Picard group of $X$ is given by

$$
\operatorname{Pic}(X)=H^{1,1}(X) \cap H^{2}(X, \mathbb{Z}) ;
$$

it classifies holomorphic line bundles on $X$. A K3 surface is projective iff there is a line bundle $L \in \operatorname{Pic}(X)$ with $L^{2}>0$ [BPV, IV.5].

The Kähler cone. Every K3 surface is Kähler. The Kähler cone

$$
C_{X} \subset H^{1,1}(X)_{\mathbb{R}}
$$

is the set of all classes represented by the symplectic forms of Kähler metrics on $X$. (Here $V_{\mathbb{R}}=\{v \in V: v=\bar{v}\}$.)

The shape of the Kähler cone can be made more explicit as follows. Let

$$
\begin{aligned}
\Delta(X) & =\left\{D \in \operatorname{Pic}(X): D^{2}=-2\right\}, \quad \text { and } \\
W(X) & =\left\{\omega \in H^{1,1}(X)_{\mathbb{R}}: \omega^{2}>0 \text { and } \omega \cdot D \neq 0 \text { for all } D \in \Delta(X)\right\} .
\end{aligned}
$$

Since the intersection form on $H^{1,1}(X)_{\mathbb{R}}$ has signature $(1,19), W(X)$ is the cone over two copies of the hyperbolic space $\mathbb{H}^{19}$, with a configuration of hyperplanes corresponding to $\Delta(X)$ deleted.

It is known that the Kähler cone $C_{X}$ coincides with a component or chamber of $W(X)$. The automorphisms of $H^{2}(X, \mathbb{Z})$ preserving the intersection form and Hodge structure act transitively on the set of chambers. (Observe that such automorphisms include the reflections $C \mapsto C+(C \cdot D) D$ through the hyperplanes defined by $D \in \Delta(X)$.)

Torelli theorem. The Torelli theorem asserts that a K3 surface is determined up to isomorphism by its Hodge structure. More precisely we have:

Theorem 2.1 Let $X$ and $Y$ be K3 surfaces, and let

$$
F: H^{2}(X, \mathbb{Z}) \rightarrow H^{2}(Y, \mathbb{Z})
$$

be an isomorphism preserving the intersection pairing. Extend $F$ to $H^{2}(X, \mathbb{C})$ by tensoring with $\mathbb{C}$; then:

1. If $F$ sends $H^{2,0}(X)$ to $H^{2,0}(Y)$, then $X$ and $Y$ are isomorphic.

2. If $F$ also sends $C_{X}$ to $C_{Y}$, then $F=f^{*}$ for a unique isomorphism $f: Y \rightarrow X$. 
Marked K3 surfaces. Next we discuss the space of all possible Hodge structures on a K3 surface.

Let $L$ be a fixed even, unimodular lattice of signature $(3,19)$. A Hodge structure on $L$ is a splitting

$$
L \otimes \mathbb{C}=H^{2,0} \oplus H^{1,1} \oplus H^{0,2}
$$

such that $\operatorname{dim} H^{2,0}=1, H^{2,0}=\overline{H^{0,2}}, H^{2,0} \oplus H^{0,2}$ has signature $(2,0)$ and $H^{1,1}=\left(H^{2,0} \oplus H^{0,2}\right)^{\perp}$. The space of Hodge structures on $L$ is parameterized by the period domain

$$
\Omega(L)=\{[\eta] \in \mathbb{P}(L \otimes \mathbb{C}): \eta \cdot \eta=0 \text { and } \eta \cdot \bar{\eta}>0\},
$$

via the correspondence

$$
H^{2,0} \oplus H^{1,1} \oplus H^{0,2}=\mathbb{C} \cdot \eta \oplus\{\eta, \bar{\eta}\}^{\perp} \oplus \mathbb{C} \cdot \bar{\eta} .
$$

The period domain is an open subset of a smooth, 20-dimensional quadric hypersurface in $\mathbb{P}^{21}$.

Now let $X$ be a K3 surface. A marking for $X$ is an isomorphism

$$
\iota: H^{2}(X, \mathbb{Z}) \cong L
$$

preserving the intersection pairing. Every K3 surface admits a marking. Two marked surfaces $\left(X_{1}, \iota_{1}\right),\left(X_{2}, \iota_{2}\right)$ are equivalent if there is an isomorphism $f: X_{1} \rightarrow X_{2}$ such that $\iota_{2}=\iota_{1} \circ f^{*}$.

Let $\mathcal{M}(L)$ be the moduli space of equivalence classes of K3 surfaces marked by $L$. The period mapping

$$
\pi: \mathcal{M}(L) \rightarrow \Omega(L) \subset \mathbb{P}(L \otimes \mathbb{C})
$$

is defined by $\pi(X, \iota)=[\iota(\eta)]$, where $\eta \neq 0$ is a holomorphic $(2,0)$-form on $X$. The image of $\pi$ lies in the period domain because $\eta \wedge \eta=0$ and $\eta \cdot \bar{\eta}>0$.

The next result complements the Torelli theorem by showing all possible Hodge structures on K3 surfaces actually arise:

Theorem 2.2 The period mapping $\pi: \mathcal{M}(L) \rightarrow \Omega(L)$ is surjective.

Note: the period mapping is not injective, because the marked Hodge structure on $H^{2}(X)$ does not uniquely determine the Kähler cone $C_{X}$. 


\section{Automorphisms of K3 surfaces}

Given a K3 surface $X$, let $\operatorname{Aut}(X)$ denote the group of biholomorphic maps $f: X \rightarrow X$.

By the Torelli theorem, the map $f \mapsto f^{*} \mid H^{2}(X)$ gives an isomorphism

$$
\operatorname{Aut}(X) \cong \operatorname{Aut}\left(H^{2}(X, \mathbb{Z}), H^{2,0}(X), C_{X}\right) .
$$

That is, $\operatorname{Aut}(X)$ acts faithfully on $H^{2}(X, \mathbb{Z})$, preserving the intersection form, the Hodge decomposition and the Kähler cone $C_{X}$; and conversely, any automorphism of $H^{2}(X, \mathbb{Z})$ preserving these three structures is induced by an automorphism of $X$.

In this section we study the eigenvalues of $f^{*} \mid H^{2}(X)$, and the synthesis of automorphisms with a given action on $H^{2}(X)$.

Algebraic numbers. A complex number $\lambda$ is algebraic if $P(\lambda)=0$ for some irreducible, monic polynomial $P(t) \in \mathbb{Q}[t]$. The degree of $\lambda$ is the degree of $P(t)$, and the roots of $P(t)$ in $\mathbb{C}$ are the conjugates of $\lambda$. If we have $P(t) \in \mathbb{Z}[t]$ then $\lambda$ is an algebraic integer; and if $\lambda^{-1}$ is also an algebraic integer, then $\lambda$ is a unit. If $\lambda$ is a root of unity, then $P(t)$ is a cyclotomic polynomial. An algebraic integer is a root of unity iff all its conjugates lie on the unit circle $S^{1} \subset \mathbb{C}$.

Salem numbers. A Salem number is a unit $\lambda>1$ whose conjugates other than $\lambda^{ \pm 1}$ lie on the unit circle. The irreducible polynomial $S(t)$ of $\lambda$ is a Salem polynomial; it has even degree, with roots of the form

$$
\left\{\lambda, \lambda^{-1}, \alpha_{1}, \bar{\alpha}_{1}, \ldots, \alpha_{d}, \bar{\alpha}_{d}\right\}
$$

where $\left|\alpha_{i}\right|=1$. (For our purposes it is natural to permit quadratic Salem numbers; these were excluded in Salem's original definition [Sa, III.3].)

A Salem trace is an algebraic integer $\tau>2$ whose other conjugates lie in $[-2,2]$; its irreducible polynomial is a Salem trace polynomial. Salem traces and Salem numbers correspond bijectively, via the relation $\tau=\lambda+\lambda^{-1}$.

Orthogonal groups. Let $\mathrm{O}(p, q) \subset \mathrm{GL}_{n}(\mathbb{R})$ denote the orthogonal group of the real quadratic form

$$
x_{1}^{2}+\cdots+x_{p}^{2}-x_{p+1}^{2}-\cdots-x_{p+q}^{2}
$$

of signature $(p, q)$. Given $T \in \mathrm{O}(p, q)$ we obtain a $T$-invariant splitting

$$
\mathbb{C}^{p+q}=\oplus E(\lambda)
$$

where $E(\lambda)=\bigcup \operatorname{Ker}(\lambda I-T)^{n}$ is the generalized $\lambda$-eigenspace of $T$. The intersection pairing is trivial between $E(\lambda)$ and $E(\mu)$ unless $\lambda \mu=1$; thus the set of eigenvalues of $T$ is invariant under $\lambda \mapsto \lambda^{-1}$. 
Lemma 3.1 A transformation $T \in \mathrm{O}(p, q)$ has at most $\min (p, q)$ eigenvalues outside the unit circle, counted with their multiplicities.

Proof. The subspace $S=\oplus_{|\lambda|>1} E(\lambda)$ is isotropic and defined over $\mathbb{R}$, so $\operatorname{dim} S \leq \min (p, q)$. But $\operatorname{dim} S$ is at least as large as the number of eigenvalues outside $S^{1}$.

Automorphisms. Now let $f: X \rightarrow X$ be an automorphism of a K3 surface, let $f^{*}=f^{*} \mid H^{2}(X, \mathbb{Z})$, and let

$$
\lambda(f)=\sup \left\{|\lambda|: \operatorname{det}\left(\lambda I-f^{*}\right)=0\right\}
$$

be the spectral radius of $f^{*}$. It is known that the topological entropy of $f$ is given by $h(f)=\log \lambda(f)$; thus $f$ has positive entropy iff $\lambda(f)>1$ [Ca1, Theorem 2.1.5] (see also [Gr], [Ym], [Frl]).

Since $f^{*}$ is invertible and preserves the integral structure on $H^{2}$, its eigenvalues are algebraic integers, in fact units.

Theorem 3.2 Either all eigenvalues of $f^{*} \mid H^{2}(X)$ are roots of unity, or there is a unique, simple eigenvalue $\lambda$ with $|\lambda|>1$, and $\lambda$ is a Salem number.

Proof. Since the conjugates of eigenvalues are again eigenvalues, if they all lie on $S^{1}$ then they are all roots of unity.

Now suppose $f^{*}$ has an eigenvalue with $|\lambda|>1$. Since $f^{*}$ stabilizes the subspace $H^{1,1}(X) \subset H^{2}(X)$ of signature $(1,19)$, it is conjugate to a transformation $T \in \mathrm{O}(2,0) \times \mathrm{O}(1,19)$. By Lemma 3.1, $f^{*}$ has at most one eigenvalue outside the unit circle, so $\lambda$ is unique, and therefore real.

As remarked above, $\lambda$ is a unit. Since $f^{*}$ preserves the Kähler cone, its $\mathrm{O}(1,19)$ part does not interchange the sheets of the light-cone in $H_{\mathbb{R}}^{1,1}$, and therefore $\lambda>1$. Applying the same reasoning to $f^{-1}$, we find that $\lambda^{-1}>0$ is the unique eigenvalue of $f^{*}$ inside the unit circle. Since all other eigenvalues of $f^{*}$ lie on the unit circle, $\lambda$ is a Salem number.

Corollary 3.3 The irreducible factors of the characteristic polynomial of $f^{*} \mid H^{2}(X)$ include at most one Salem polynomial; and the remaining factors are cyclotomic.

Synthesis of automorphisms. The next theorem provides the key to building examples of K3 surface automorphisms. It reduces the construction of automorphisms to a problem in integral quadratic forms. It also represents the first step towards determining which Salem numbers can arise as $\lambda(f)$. 
Theorem 3.4 (Synthesis) Let $F: L \rightarrow L$ be an automorphism of an even, unimodular lattice of signature $(3,19)$. Suppose $S(t)=\operatorname{det}(t I-F)$ is a Salem polynomial. Then there is:

- A K3 surface automorphism $f: X \rightarrow X$, and

- A marking $\iota: H^{2}(X, \mathbb{Z}) \rightarrow L$, such that $F=\iota \circ f^{*} \circ \iota^{-1}$.

Proof. Since $F$ has only two eigenvalues off the unit circle, while the signature of $L$ is $(3,19)$, there exists an eigenvector $\eta \in L \otimes \mathbb{C}$ such that $T(\eta)=\delta \eta,|\delta|=1$ and $\eta \cdot \bar{\eta}>0$. By surjectivity of the period mapping (Theorem 2.2), there exists a K3 surface $X$ and a marking $\iota: H^{2}(X, \mathbb{Z}) \rightarrow L$ such that $\iota\left(H^{2,0}(X)\right)=\mathbb{C} \cdot \eta$.

Let $T: H^{2}(X, \mathbb{Z}) \rightarrow H^{2}(X, \mathbb{Z})$ be the automorphism given by $T=$ $\iota^{-1} \circ F \circ \iota$. Then $T$ respects the intersection paring and the Hodge structure on $H^{2}(X)$, and its characteristic polynomial is also $S(t)$.

We claim that $\operatorname{Pic}(X)=0$. Indeed, since $S(t)$ is irreducible, $T$ has no proper rational invariant subspace, and thus $H^{1,1}(X) \cap H^{2}(X, \mathbb{Z})=(0)$. In particular, $\Delta(X)=\emptyset$, so the Kähler cone $C_{X} \subset H^{1,1}(X)_{\mathbb{R}}$ is simply one of the two components of the space $W(X)=\left\{\omega: \omega^{2}>0\right\}$.

Since the leading eigenvalue of $T$ is a Salem number $\lambda>1, T$ does not interchange the components of $W(X)$, and therefore $T\left(C_{X}\right)=C_{X}$. By the Torelli theorem (Theorem 2.1), there is a unique automorphism $f: X \rightarrow X$ such that $f^{*} \mid H^{2}(X, \mathbb{Z})=T$.

Remarks. The marked K3 surface $X$ constructed above is unique up to complex conjugation. Note that $X$ is non-projective, since $\operatorname{Pic}(X)=(0)$.

The determinant $\boldsymbol{\delta}(\boldsymbol{f})$. Let $\eta \neq 0$ be a holomorphic $(2,0)$-form on $X$. Since $\operatorname{dim} H^{2,0}(X)=1, \eta$ is always an eigenvector for $f^{*}$. Let

$$
\delta(f)=\operatorname{Tr} f^{*} \mid H^{2,0}(X)=f^{*} \eta / \eta
$$

denote the corresponding eigenvalue. The norm $\eta \cdot \bar{\eta}>0$ is preserved by $f$, so we have $|\delta(f)|=1$.

Now suppose $p \in X$ is a fixed-point of $f$, and let $D f_{p}: T_{p} X \rightarrow T_{p} X$ denote the complex derivative. Since $\eta(p)$ lies in $\wedge^{2} T_{p}^{*}(X)$, we have

$$
\delta(f)=\operatorname{det} D f_{p} .
$$

In particular, $\operatorname{det} D f_{p}$ is the same at all fixed-points. For this reason, we call $\delta(f)$ the determinant of $f$.

We conclude this section with two results about the eigenvalue $\delta(f)$. 
Theorem 3.5 If $f$ is an automorphism of a projective $K 3$ surface $X$, then $\delta(f)$ is a root of unity.

Proof. Since $X$ is projective, there is a $D \in \operatorname{Pic}(X) \subset S$ with $D^{2}>$ 0 . The subspace $H^{1,1}(X) \cap D^{\perp}$ is negative-definite, with signature $(0,19)$, and contains $\operatorname{Pic}(X) \cap D^{\perp}$. Thus the intersection form on $\operatorname{Pic}(X) \otimes \mathbb{R}$ has signature $(1, n)$ for some $n, 0 \leq n \leq 19$.

Consequently, the rational $f^{*}$-invariant subspace

$$
S=\operatorname{Pic}(X)^{\perp} \supset H^{2,0}(X) \oplus H^{0,2}(X)
$$

has signature $(2,19-n)$. Now $f^{*} \mid S$ preserves the signature $(2,0)$-subspace on the right, so it is conjugate to an element of $\mathrm{O}(2) \times \mathrm{O}(19-n)$. Thus all eigenvalues of $f^{*} \mid S$, including $\delta(f)$, lie on the unit circle. But $f^{*} \mid S$ also preserves the lattice $S \cap H^{2}(X, \mathbb{Z})$, so its characteristic polynomial lies in $\mathbb{Z}[t]$, and therefore its eigenvalues are roots of unity.

Theorem 3.6 Up to isomorphism, there are only countably many pairs $(X, f)$ where $\delta(f)$ is not a root of unity.

Proof. Assume $\delta=\delta(f)$ is not a root of unity. By Corollary 3.3, $\delta$ has multiplicity one as a root of the characteristic polynomial for $f^{*} \mid H^{2}(X, \mathbb{Z})$. Thus $H^{2,0}(X)$ is an eigenspace for $f^{*}$, and therefore $f^{*}$ determines the Hodge structure on $H^{2}(X)$ up to finitely many choices. By the Torelli theorem, the Hodge structure on $H^{2}(X)$ together with $f^{*} \mid H^{2}(X)$ determines $(X, f)$ up to isomorphism. Since there are only countably many possibilities for $f^{*} \mid H^{2}(X, \mathbb{Z})$, there are only countably many possibilities for $(X, f)$.

In $\S 4$ we will construct countably many examples where $\delta(f)$ is not a root of unity. The invariant $\delta(f)$ is studied on more general manifolds in [Bv1], which includes a version of Theorem 3.5.

\section{Ergodic dynamics on Kummer surfaces}

Every K3 surface $X$ carries a natural probability measure $\mu$, defined by

$$
\mu(A)=\int_{A} \eta \wedge \bar{\eta}
$$

where $\eta$ is a holomorphic $(2,0)$-form on $X$, scaled so $\mu(X)=1$. All automorphisms of $X$ preserve the measure $\mu$. 
An automorphism $f: X \rightarrow X$ is ergodic if every $f$-invariant Borel set satisfies $\mu(A)=0$ or 1 . It is mixing if any pair of Borel sets satisfy

$$
\mu\left(f^{n}(A) \cap B\right) \rightarrow \mu(A) \mu(B)
$$

as $|n| \rightarrow \infty$. Mixing implies ergodicity.

In this section we discuss an elementary construction of mixing automorphism of K3 surfaces, using complex tori. The same construction yields automorphisms where $\delta(f)$ is not a root of unity. For example we will show:

Theorem 4.1 For every $a=0,1,2 \ldots$, there exists a mixing automorphism of a K3 surface $f: X \rightarrow X$ such that $\lambda(f)$ and $\delta(f)$ are roots of the Salem polynomial $S(t)=t^{6}-a t^{5}-t^{4}+(2 a-1) t^{3}-t^{2}-a t+1$.

Complex tori. An $n$-dimensional complex torus is a compact complex Lie group of the form $Y=\mathbb{C}^{n} / \Lambda$, where $\Lambda$ is a lattice in $\mathbb{C}^{n}$. As in the case of K3 surfaces, the canonical bundle of $Y$ is trivial; $H^{n, 0}(Y)$ is 1-dimensional, spanned by the holomorphic form $\xi=d z_{1} \wedge \cdots \wedge d z_{n}$.

Let $\operatorname{Aut}(Y)$ denote the automorphisms of $Y$ as a complex Lie group. Elements $F \in \operatorname{Aut}(Y)$ correspond bijectively to linear maps

$$
\widetilde{F}: \mathbb{C}^{n} \rightarrow \mathbb{C}^{n}
$$

satisfying $\widetilde{F}(\Lambda)=\Lambda$. The eigenvalues $\left(\lambda_{1}, \ldots, \lambda_{n}\right)$ of $\widetilde{F}$ coincide with the eigenvalues of $F^{*} \mid H^{1}(Y)$. Defining $\lambda(F)$ as the spectral radius of $F^{*} \mid H^{*}(Y)$ and $\delta(F)$ as $F^{*} \xi / \xi$, one can easily check that:

$$
\begin{aligned}
& \lambda(F)=\prod\left\{\left|\lambda_{i}\right|^{2}:\left|\lambda_{i}\right| \geq 1\right\} \quad \text { and } \\
& \delta(F)=\lambda_{1} \lambda_{2} \cdots \lambda_{n} .
\end{aligned}
$$

We have $|\delta(F)|=|\operatorname{det}(F)|=1$.

The measure $\xi \wedge \bar{\xi}$ on $Y$ is $\operatorname{Aut}(Y)$-invariant. Using Fourier analysis on the space of square-integrable functions $L^{2}(Y)$, one finds (cf. [Man, Chapter II, 3.1, 8.5 and 8.6]):

Theorem 4.2 An automorphism $F \in \operatorname{Aut}(Y)$ is ergodic iff $F$ is mixing iff no eigenvalue of $F^{*} \mid H^{1}(Y)$ is a root of unity.

Kummer surfaces. Now let $Y=\mathbb{C}^{2} / \Lambda$ be a 2-dimensional complex torus, and let $\iota: Y \rightarrow Y$ be the involution $\iota(y)=-y$. Blowing up the 16 double points of the quotient space $Y / \iota$, we obtain the Kummer surface $X=\kappa(Y)$; 
it comes equipped with a degree 2 rational map $Y \rightarrow X$. The complex manifold $X$ is simply-connected, and the form $\xi=d z_{1} \wedge d z_{2}$ on $Y$ descends to a nowhere-vanishing holomorphic $(2,0)$-form $\eta$ on $X$; thus $X$ is a K3 surface.

Since every $F \in \operatorname{Aut}(Y)$ commutes with the involution $\iota$, it gives rise to an automorphism $f=\kappa(F) \in \operatorname{Aut}(X)$.

Theorem 4.3 Let $F$ be an automorphism of a complex 2-torus $Y$, and $f$ the corresponding automorphism of the Kummer surface $X=\kappa(Y)$. Then

$$
\delta(F)=\delta(f), \quad \lambda(F)=\lambda(f),
$$

and $F$ is mixing $\Longleftrightarrow f$ is mixing.

Proof. The rational map $Y \rightarrow X$ determines a natural isomorphism $H^{2}(X)=H^{2}(Y) \oplus \mathbb{C}^{Y[2]}$, where $Y[2] \subset Y$ denotes the 16 points of order 2 [BPV, VIII.5]. Under this isomorphism, the action of $f^{*} \mid H^{2}(Y)$ corresponds to the action of $F^{*} \mid H^{2}(Y)$ together with the action of $F$ by permutations on $Y[2]$; thus $\lambda(f)=\lambda(F)$. Moreover $H^{2,0}(X)$ corresponds to $H^{2,0}(Y)$, so $\delta(f)=\delta(F)$. For the assertions on mixing, use the fact that $L^{2}(X)$ is canonically identified with the set of even functions in $L^{2}(Y)$.

Example: $\mathbf{S L}_{\mathbf{2}}(\mathbb{Z})$. As a first example, let $Y=E \times E$ where $E=\mathbb{C} / \Lambda$ is a 1-dimensional complex torus. Then each matrix $A=\left(\begin{array}{ll}a & b \\ c & d\end{array}\right) \in \mathrm{SL}_{2}(\mathbb{Z})$ acts on $Y$ by

$$
F_{A}\left(e_{1}, e_{2}\right)=\left(a e_{1}+b e_{2}, c e_{1}+d e_{2}\right),
$$

and $F_{A}$ is mixing iff $A$ has no eigenvalues on $S^{1}$. Thus each matrix $A \in$ $\mathrm{SL}_{2}(\mathbb{Z})$ with $|\operatorname{Tr} A|>2$ gives rise to a mixing automorphism $f=\kappa\left(F_{A}\right)$ on the Kummer surface $X=\kappa(E \times E)$.

Synthesis of dynamics. These $\mathrm{SL}_{2}(\mathbb{Z})$ examples satisfy $\delta(f)=1$. To obtain a greater variety of values for $\delta(f)$, we will synthesize an automorphism from the desired characteristic polynomial for $F^{*} \mid H^{1}(Y)$.

Theorem 4.4 Let $P(t)=t^{2 n}+a_{1} t^{2 n-1}+\cdots+a_{2 n-1} t+1 \in \mathbb{Z}[t]$ be $a$ polynomial whose roots occur in conjugate pairs

$$
\left\{\alpha_{1}, \bar{\alpha}_{1}, \ldots, \alpha_{n}, \bar{\alpha}_{n}\right\}
$$

Then there is a complex torus $Y$ and an $F \in \operatorname{Aut}(Y)$ such that

$$
\begin{aligned}
P(t) & =\operatorname{det}\left(t I-F^{*} \mid H^{1}(Y)\right) \quad \text { and } \\
\delta(F) & =\alpha_{1} \alpha_{2} \cdots \alpha_{n} .
\end{aligned}
$$


Proof. Choose a semisimple transformation $T \in \mathrm{SL}_{2 n} \mathbb{Z}$ whose characteristic polynomial is $P(t)$; this is possible because $P(0)=1$. Since the roots of $P(t)$ occur in conjugate pairs, we can find a basis $\left(v_{i}, \bar{v}_{i}\right)_{1}^{n}$ for $\mathbb{C}^{2 n}$ such that such that $T v_{i}=\alpha_{i} v_{i}$. The pairs of conjugate eigenvectors determine a splitting $\mathbb{R}^{2 n}=\oplus_{1}^{n} E_{i}$ where $E_{i} \otimes \mathbb{C}=\mathbb{C} v_{i} \oplus \mathbb{C} \bar{v}_{i}$. We can then choose an $\mathbb{R}$-linear isomorphism $\phi: \mathbb{R}^{2 n} \rightarrow \mathbb{C}^{n}$ sending each $E_{i}$ to a coordinate axis, such that $\phi(T v)=\widetilde{F}(\phi(v))$, where $\widetilde{F}\left(z_{1}, \ldots, z_{n}\right)=\left(\alpha_{1} z_{1}, \ldots, \alpha_{n} z_{n}\right)$. Setting $\Lambda=\phi\left(\mathbb{Z}^{2 n}\right)$ and $Y=\mathbb{C}^{n} / \Lambda$, and letting $\widetilde{F}$ descend to $F \in \operatorname{Aut}(Y)$, we obtain the required automorphism.

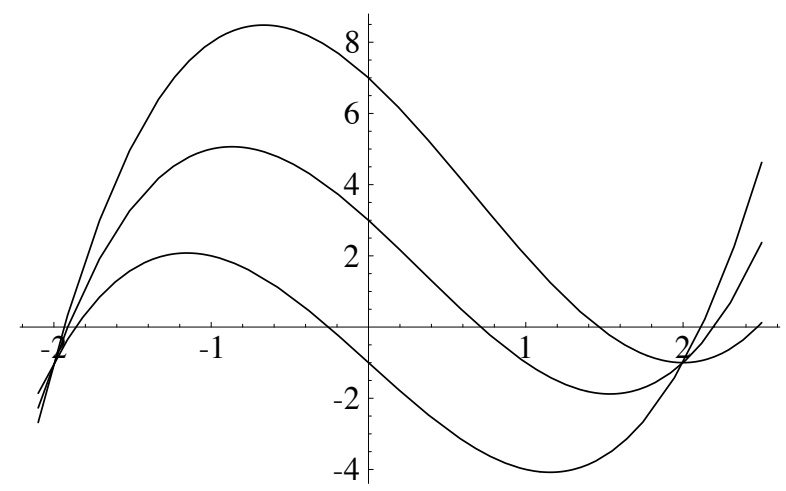

Figure 3. Salem trace polynomials $R(t)=(t-a)\left(t^{2}-4\right)-1$ for $a=0,1,2$.

Examples: degree 6 Salem numbers. For any integer $a \geq 0$, it is easy to check that

$$
P(t)=t^{4}+a t^{2}+t+1
$$

has only complex roots, say $\{\alpha, \beta, \bar{\alpha}, \bar{\beta}\}$ with $|\alpha|>|\beta|$. By the preceding result, there is a complex 2-torus $Y$ and an automorphism $F \in \operatorname{Aut}(Y)$ such that

$$
\delta(F)=\alpha \beta, \quad \lambda(F)=|\alpha|^{2},
$$

and $P(t)$ is the characteristic polynomial of $F^{*} \mid H^{1}(Y)$.

The products of pairs of distinct roots of $P(t)$ give the roots of the characteristic polynomial

$$
S(t)=t^{6}-a t^{5}-t^{4}+(2 a-1) t^{3}-t^{2}-a t+1
$$

of $F^{*} \mid H^{2}(Y)=\wedge^{2} H^{1}(Y)$. Thus $\delta(F)$ and $\lambda(F)$ are roots of $S(t)$. Similarly, $\tau=\lambda(F)+\lambda(F)^{-1}>2$ is a root of the cubic polynomial

$$
R(t)=(t-a)\left(t^{2}-4\right)-1 .
$$


See Figure 3. (The formulas for $S(t)$ and $R(t)$ come from a straightforward calculation with determinants and the companion matrix of $P(t)$.)

Since $R(-2)=R(2)=-1$ while $R(-1)>0$, the roots of $R(t)$ other than $\tau$ lie in the interval $[-2,2]$, and since $R(n) \neq 0$ for $n \in \mathbb{Z}, R(t)$ is irreducible. Thus $R(t)$ is a Salem trace polynomial. Therefore $\lambda(F)$ is a sextic Salem number, and $S(t)$ is a Salem polynomial.

Proof of Theorem 4.1. In the examples just discussed, pass to the Kummer surface $X=\kappa(Y)$.

Note that in these examples, $\delta(F)=\alpha \beta$ is not a root of unity, since it is conjugate to $\lambda(F)=|\alpha|^{2}>1$.

Lattès examples. We remark that the automorphisms of Kummer surfaces discussed above are higher-dimensional relatives of the Lattès examples of rational maps $f: \mathbb{P}^{1} \rightarrow \mathbb{P}^{1}$. To construct $f$, start with an endomorphism $F(y)=n y$ of a complex 1-torus $Y=\mathbb{C} / \Lambda$. Observing that the quotient $X=Y /(y \sim-y)$ is isomorphic to $\mathbb{P}^{1}$, one finds that $F$ descends a rational map $f: \mathbb{P}^{1} \rightarrow \mathbb{P}^{1}$ of degree $n^{2}$, and $f$ is ergodic if $n>1$. These are among the simplest rational maps with Julia set the whole Riemann sphere [Mil, $\S 7],[\mathrm{Mc}, \S 3.5]$.

Towards Siegel disks. As discussed above, the ergodicity of an automorphism of a complex torus can be detected by its action on cohomology: $F$ is ergodic iff the eigenvalues of $F^{*} \mid H^{1}(Y)$ include no roots of unity.

Similarly, the next three sections develop a cohomological criterion for the existence of a Siegel disk on a K3 surface, based on the action of $f^{*}$ on $H^{2}(X)$.

\section{$5 \quad$ Siegel disks and transcendence theory}

This section applies transcendence theory to establish the existence of Siegel disks in an arithmetic setting. The main result is:

Theorem 5.1 Let $X$ be a complex $n$-manifold, and let $f: X \rightarrow X$ be an holomorphic map fixing $p \in X$. If the eigenvalues $\left(\lambda_{i}\right)$ of $D f_{p}$ are algebraic, multiplicatively independent and satisfy $\left|\lambda_{i}\right|=1$, then $f$ has a Siegel disk at $p$.

The proof uses results originally developed to address Hilbert's seventh problem, on the transcendence of numbers like $2^{\sqrt{2}}$. 
Definitions. Let $\left(\lambda_{1}, \ldots, \lambda_{n}\right)$ be nonzero complex numbers. We say $\left(\lambda_{i}\right)$ are multiplicatively independent if the only solution to

$$
\lambda_{1}^{k_{1}} \cdots \lambda_{n}^{k_{n}}=1
$$

with $k=\left(k_{1}, \ldots, k_{n}\right) \in \mathbb{Z}^{n}$ is $k=0$. The numbers $\left(\lambda_{i}\right)$ are jointly Diophantine if there exist $C, M>0$ such that for all integral exponents $\left(k_{1}, \ldots, k_{n}\right) \in$ $\mathbb{Z}^{n}$, not all zero, we have

$$
\left|\lambda_{1}^{k_{1}} \cdots \lambda_{n}{ }^{k_{n}}-1\right|>C\left(\max \left|k_{i}\right|\right)^{-M}>0 .
$$

Theorem 5.2 Let $\left(\lambda_{1}, \cdots, \lambda_{n}\right)$ be multiplicatively independent algebraic numbers on $S^{1}$. Then $\left(\lambda_{1}, \ldots, \lambda_{n}\right)$ are jointly Diophantine.

Proof. By multiplicative independence, the numbers $\left(2 \pi i, \log \lambda_{1}, \ldots, \log \lambda_{n}\right)$ are linearly independent over $\mathbb{Q}$. Using the Gel'fond-Baker method, Fel'dman shows that linear independence over $\mathbb{Q}$ implies linear independence over any number field, in the following effective sense [Fe]: for any algebraic numbers $\left(k_{i}\right)$ not all zero, we have

$$
\left|k_{0} 2 \pi i+k_{1} \log \lambda_{1}+\cdots+k_{n} \log \lambda_{n}\right|>\exp (-M(d+\log H)),
$$

where $d$ is the degree of the field $\mathbb{Q}\left[k_{0}, \ldots, k_{n}, \lambda_{1}, \ldots, \lambda_{n}\right], M=M\left(\lambda_{i}, d\right)$ is a constant depending only on the numbers $\left(\lambda_{i}\right)$ and $d, H=\max H\left(k_{i}\right)$, and the height $H(k)=\sum\left|a_{j}\right|$ if $p(k)=0$ where $p(x)=\sum_{0}^{s} a_{j} x^{j}$ is an irreducible polynomial with relatively prime coefficients $a_{j} \in \mathbb{Z}$.

For $k_{i} \in \mathbb{Z}$ we have $H=\max \left|k_{i}\right|$, and $M$ depends only on $\left(\lambda_{i}\right)$; therefore

$$
\exp (-M(d+\log H))=C\left(\max \left|k_{i}\right|\right)^{-M}
$$

for some $C>0$. Thus (5.2) implies the Diophantine condition (5.1).

Remark. Taking $\lambda_{1}^{k_{1}}=\alpha^{\beta}$, Fel'dman's bound (5.2) implies the Gel'fondSchneider theorem: $\alpha^{\beta}$ is transcendental for all algebraic numbers $\alpha \neq 0,1$ and $\beta \notin \mathbb{Q}$.

Siegel disks. Let $F: \mathbb{C}^{n} \rightarrow \mathbb{C}^{n}$ be a linear map of the form

$$
F\left(z_{1}, \ldots, z_{n}\right)=\left(\lambda_{1} z_{1}, \ldots, \lambda_{n} z_{n}\right)
$$

with $\left|\lambda_{i}\right|=1$. We say $F$ is an irrational rotation if the numbers $\left(\lambda_{i}\right)$ are multiplicatively independent; equivalently, if the orbits of $F$ on $\left(S^{1}\right)^{n}$ are dense. 
Now let $f: X \rightarrow X$ be a holomorphic endomorphism of a complex $n$ manifold, and suppose $f(p)=p$. We say $f$ has a Siegel disk $U$, centered at $p$, if there is a neighborhood $U$ of $p$ and an analytic isomorphism to a polydisk,

$$
\phi:(U, p) \rightarrow\left(\Delta^{n}, 0\right),
$$

sending the action of $f \mid U$ to the action of an irrational rotation $F \mid \Delta^{n}$.

Here is a local criterion for the existence of a Siegel disk.

Theorem 5.3 If the derivative $D f_{p}: T_{p} X \rightarrow T_{p} X$ has jointly Diophantine eigenvalues $\left(\lambda_{1}, \ldots, \lambda_{n}\right) \in\left(S^{1}\right)^{n}$, then $f$ has a Siegel disk at $p$.

The proof is due to Siegel for $n=1$ and to Sternberg for $n>1$ [St, p. 465].

Proof of Theorem 5.1: Combine the results of Fel'dman and Sternberg.

Notes. See [Ti] and [Her] for more on the Gel'fond-Baker method and analytic linearization respectively. In Theorem 5.3, (5.1) can be replaced by the weaker condition $\inf _{j}\left|\lambda_{1}^{k_{1}} \cdots \lambda_{n}{ }^{k_{n}}-\lambda_{j}\right|>C\left|k_{1}+\cdots+k_{n}\right|^{-M}$ for all $\left(k_{1}, \ldots, k_{n}\right) \in \mathbb{Z}^{n}$ with $k_{i} \geq 0$ and $\sum k_{i} \geq 2$.

\section{Holomorphic Lefschetz numbers}

Let $f: X \rightarrow X$ be an automorphism of a complex manifold with a unique fixed-point $p$. In this section we show the derivative $D f_{p}$ is determined by the action of $f^{*}$ on $H^{r, s}(X)$. In the special case of K3 surfaces, we obtain:

Theorem 6.1 Let $f: X \rightarrow X$ be an automorphism of a K3 surface with a unique transverse fixed-point $p$. Then we have

$$
\operatorname{Tr} D f_{p}=\frac{1+\delta+\delta^{2}}{1+\delta}
$$

where $\delta=\delta(f)$.

The Atiyah-Bott fixed-point theorem. Let $f: X \rightarrow X$ be a holomorphic automorphism of a compact complex manifold $X$ with real dimension $n$. Then $f$ acts on the singular cohomology groups $H^{q}(X), 0 \leq q \leq n$, as well as the Dolbeault cohomology groups $H^{r, s}(X)$. We define the Lefschetz number of $f$ by

$$
L(f)=\sum_{q=0}^{n}(-1)^{q} \operatorname{Tr} f^{*} \mid H^{q}(X),
$$


and the $r$ th holomorphic Lefschetz number of $f$ by

$$
L^{r}(f)=\sum_{s=0}^{n}(-1)^{s} \operatorname{Tr} f^{*} \mid H^{r, s}(X) .
$$

It is known that $L(f)=\sum(-1)^{r} L^{r}(f)$, and if $f$ has a finite set of fixed-points then their number, counted with multiplicity, is $L(f)$.

We say $f$ has transverse fixed-points if the graph of $f$ is transverse to the diagonal in $X \times X$. In this case, the set of fixed-points $p \in X$ is finite, every fixed-point has multiplicity one, and by transversality the complex derivative

$$
D f_{p}: T_{p}(X) \rightarrow T_{p}(X)
$$

satisfies $\operatorname{det}\left(I-D f_{p}\right) \neq 0$. The Atiyah-Bott fixed-point theorem states [AB, $(4.9-4.10)]$ :

Theorem 6.2 If $f$ has transverse fixed-points, then

$$
L^{r}(f)=\sum_{f(p)=p} \frac{\operatorname{Tr} \wedge^{r} D f_{p}}{\operatorname{det}\left(I-D f_{p}\right)} .
$$

Corollary 6.3 If $f$ has a unique, transverse fixed-point $p$, then we have

$$
\operatorname{det}\left(t I-D f_{p}\right)=L^{0}(f)^{-1} \sum_{0}^{n}(-1)^{r} L^{r}(f) t^{n-r} .
$$

Proof. Apply the formula $\operatorname{det}(I-A)=\sum(-1)^{r} \operatorname{Tr} \wedge^{r} A$.

Proof of Theorem 6.1. From the Atiyah-Bott formula with $r=2$ and the fact that $\delta=\operatorname{Tr} f^{*} \mid H^{2,0}(X)=\operatorname{det} D f_{p}$, we obtain

$$
L^{2}(f)=1+\delta=\frac{\operatorname{Tr} \wedge^{2} D f_{p}}{\operatorname{det}\left(I-D f_{p}\right)}=\frac{\delta}{1-\operatorname{Tr} D f_{p}+\delta},
$$

which gives (6.1). 


\section{$7 \quad$ Siegel disks on K3 surfaces}

With the results of the preceding sections in hand, we can now establish a cohomological criterion for the existence of a Siegel disk on a K3 surface.

Theorem 7.1 Let $f: X \rightarrow X$ be an automorphism of a K3 surface. Suppose that:

1. The characteristic polynomial of $f^{*} \mid H^{2}(X)$ is a Salem polynomial;

2. $\operatorname{Tr} f^{*} \mid H^{2}(X)=-1$;

3. The determinant $\delta=\delta(f)$ satisfies $\tau=\delta+\delta^{-1}>1-2 \sqrt{2}$; and

4. The algebraic integer $\tau$ has a conjugate $\tau^{\prime}<1-2 \sqrt{2}$.

Then $f$ has a unique fixed-point $p$, and $p$ is the center of a Siegel disk.

We begin by remarking that Siegel disks are rare.

Theorem 7.2 An automorphism of a projective K3 surface never has a Siegel disk. Moreover, there are at most countably many K3 surface automorphisms $(X, f)$ with Siegel disks, up to isomorphism.

Proof. Suppose $f$ has a Siegel disk centered at $p$, and let $\alpha, \beta$ be the eigenvalues of $D f_{p}$. Since $\alpha$ and $\beta$ are multiplicatively independent, $\delta(f)=$ $\alpha \beta$ is not a root of unity. By Theorems 3.5 and 3.6, $X$ is non-projective, and its Hodge structure is rigid, limiting $(X, f)$ to a countable set.

Resonances. Theorem 7.2 does not rule out the possibility of a 'resonant' rotation domain on a projective $\mathrm{K} 3$ surface. That is, $f: X \rightarrow X$ might conceivably have a linearizable fixed-point $p$ where the eigenvalues $\lambda_{1}, \lambda_{2}$ of $D f_{p}$ lie on $S^{1}$ but are not multiplicatively independent. Nevertheless, such a fixed-point seems unlikely to exist. Generically, a resonant fixed-point is not even formally linearizable; and if $\lambda_{1}^{k_{1}} \lambda_{2}^{k_{2}}=1$, then $z_{1}^{k_{1}} z_{2}^{k_{2}}$ is invariant under the linear map $\left(z_{1}, z_{2}\right) \mapsto\left(\lambda_{1} z_{1}, \lambda_{2} z_{2}\right)$, and thus $f$ must preserve a pencil of analytic curves near $p$.

We remark that Siegel disks can exist only for automorphisms of positive entropy.

Theorem 7.3 If $f: X \rightarrow X$ is a K3 surface automorphism with a Siegel disk, then $\lambda(f)>1$.

Proof. Since $\delta(f)$ is an eigenvalue of $f^{*} \mid H^{2}(X)$ and not a root of unity, there must also be an eigenvalue off the unit circle. 
To obtain a Siegel disk we need conditions for the existence of a fixedpoint $p$ and for multiplicative independence of the eigenvalues of $D f_{p}$.

Theorem 7.4 Suppose $\operatorname{Tr} f^{*} \mid H^{2}(X)=-1$ and $\left(I-f^{*}\right)$ is invertible on $H^{1,1}(X)$. Then $f$ has a unique, transverse fixed-point $p \in X$.

Proof First note that fixed-points of $f$ are isolated; otherwise the locus $f(p)=p$ would contain a 1-dimensional analytic cycle, representing an $f^{*}$ invariant class $C \in H^{(1,1)}(X)$. Since every K3 surface is Kähler, we would have $C \neq 0$, contrary to our hypothesis on $I-f^{*}$.

Next, recall that every isolated fixed-point of a holomorphic map has positive multiplicity. Since $L(f)=2+\operatorname{Tr} f^{*} \mid H^{2}(X)=1, f$ has a unique fixed-point $p$, of multiplicity one and hence transverse.

Lemma 7.5 Let $\delta$ be a root of a Salem polynomial with $|\delta|=1$, let $\tau=$ $\delta+1 / \delta$ and let $\tau^{\prime}$ be a conjugate of $\tau$. Suppose we have

$$
\tau^{\prime}<1-2 \sqrt{2}<\tau
$$

Then the roots $(\alpha, \beta)$ of the simultaneous equations

$$
\alpha \beta=\delta \quad \text { and } \quad \alpha+\beta=\frac{1+\delta+\delta^{2}}{1+\delta}
$$

are multiplicatively independent, with $|\alpha|=|\beta|=1$.

Proof. Since $\alpha \beta / \delta=1$ and $|\delta|=1$, we have $\alpha, \beta \in S^{1} \Longleftrightarrow(\alpha+\beta)^{2} / \delta \in$ $[0,4]$. By $(7.1)$, this condition is the same as

$$
\frac{\left(1+\delta+\delta^{2}\right)^{2}}{\delta(1+\delta)^{2}}=\frac{\left(\delta^{-1}+1+\delta\right)^{2}}{\left(\delta^{-1}+2+\delta\right)}=\frac{(\tau+1)^{2}}{\tau+2} \in[0,4],
$$

which holds iff $\tau \in(1-2 \sqrt{2}, 1+2 \sqrt{2})$. By assumption $\tau>1-2 \sqrt{2}$, and we have $\tau<2<1+2 \sqrt{2}$ because $|\delta|=1$. Thus $|\alpha|=|\beta|=1$.

By Galois theory, there exist conjugates $\left(\alpha^{\prime}, \beta^{\prime}, \delta^{\prime}\right)$ of $(\alpha, \beta, \delta)$ also satisfying (7.1), with $\delta^{\prime}+1 / \delta^{\prime}=\tau^{\prime}$. Since $\tau^{\prime}<2$ and $\delta^{\prime}$ is a root of a Salem polynomial, we have $\left|\delta^{\prime}\right|=1$. But now $\tau^{\prime} \notin(1-2 \sqrt{2}, 1+2 \sqrt{2})$, so we have $\left|\alpha^{\prime}\right|=\left|\beta^{\prime}\right|^{-1} \neq 1$.

Now suppose $\alpha^{i} \beta^{j}=1$. Then $\left(\alpha^{\prime}\right)^{i}\left(\beta^{\prime}\right)^{j}=1$ as well, and thus $i=j$. Therefore $(\alpha \beta)^{i}=\delta^{i}=1$. Since $\delta$ satisfies a Salem polynomial, it is not a root of unity, and thus $i=0$. Therefore $\alpha$ and $\beta$ are multiplicatively independent. 
Proof of Theorem 7.1. By Theorem 7.4, $f$ has a unique fixed-point $p \in X$. Via Theorem 6.1, the Atiyah-Bott formula implies the eigenvalues $(\alpha, \beta)$ of $D f_{p}$ satisfy $(7.1)$ with $\delta=\delta(f)=\operatorname{det} D f_{p}$. Since $\delta(f)$ is an algebraic number, so are $\alpha$ and $\beta$. The preceding Lemma shows $\alpha$ and $\beta$ lie on the unit circle and are multiplicatively independent. Theorem 5.1 states that such algebraic numbers satisfy the Diophantine condition for linearization, so $f$ has a Siegel disk at $p$.

\section{Lattices in number fields}

In this section we use number theory to construct lattice automorphisms with given characteristic polynomials. Via the Torelli theorem, these lattice automorphisms will give rise to K3 surface automorphisms.

To motivate the construction, suppose $F: B \rightarrow B$ is an automorphism of a lattice, with irreducible characteristic polynomial $s(y)=\operatorname{det}(y I-F) \in$ $\mathbb{Z}[y]$. Since the pairing between the $\lambda$ and $\mu$ eigenspaces of $F$ is nontrivial iff $\lambda \mu=1$, the eigenvalues of $F$ occur in reciprocal pairs. Thus $s(y)$ has degree $2 d$, and there is a degree $d$ polynomial $r(x) \in \mathbb{Z}[x]$ whose roots have the form $\tau=\lambda+\lambda^{-1}$ as $\lambda$ ranges over the roots of $s(y)$.

In this section we reverse the discussion. Starting from a degree $d$ polynomial $r(x)$ such that the associated degree $2 d$ polynomial $s(y)$ is irreducible, we construct a lattice $B$ and an automorphism $F: B \rightarrow B$ with characteristic polynomial $s(y)$. The construction can be twisted by a unit $u \in \mathbb{Z}[x] /(r(x))$, yielding lattices $B(u)$ with a range of different of signatures.

Discriminants. Let $L \cong \mathbb{Z}^{n}$ be a lattice with inner product $\langle x, y\rangle_{L} \in \mathbb{Z}$. Given a basis $\left(e_{i}\right)_{1}^{n}$ for $L$, the discriminant of $L$ is given by

$$
\operatorname{disc}(L)=\operatorname{det}\left(\left\langle e_{i}, e_{j}\right\rangle\right)
$$

it is independent of the choice of basis. The inner product allows us to regard $L$ as a subgroup of $L^{*}=\operatorname{Hom}(L, \mathbb{Z})$, via $x(y)=\langle x, y\rangle_{L}$, and we have $|\operatorname{disc}(L)|=\left|L^{*} / L\right|$. Thus $L$ is unimodular iff $|\operatorname{disc}(L)|=1$.

A symmetric linear embedding $Q: L \rightarrow L$ determines a new lattice $L(Q)$ with the same underlying free abelian group as $L$, but with the new inner product

$$
\langle x, y\rangle_{L(Q)}=\langle Q x, y\rangle_{L}
$$

We have $\operatorname{disc}(L(Q))=\operatorname{det}(Q) \cdot \operatorname{disc}(L)$.

Trace forms. Lattices occur naturally in number rings. Let $r(x) \in \mathbb{Z}[x]$ be a degree $d$ irreducible monic polynomial with roots $\left(x_{i}\right)_{1}^{d}$ in $\mathbb{C}$. Let $A$ be 
the integral domain $\mathbb{Z}[x] / r(x)$ and let $k$ be its field of fractions. Define an inner product on $A$ by

$$
\left\langle f_{1}, f_{2}\right\rangle_{A}=\operatorname{Tr}_{\mathbb{Q}}^{k}\left(\frac{f_{1}(x) f_{2}(x)}{r^{\prime}(x)}\right)=\sum_{1}^{d}\left(\frac{f_{1}\left(x_{i}\right) f_{2}\left(x_{i}\right)}{r^{\prime}\left(x_{i}\right)}\right)
$$

(where $\left.r^{\prime}(x)=d r / d x\right)$.

As was known to Euler, this inner product takes values in $\mathbb{Z}$ and makes $A$ into a unimodular lattice. To prove this, one can use the residue theorem to compute:

$$
\begin{aligned}
\left\langle 1, x^{n}\right\rangle_{A} & =\sum \operatorname{Res}\left(x^{n} d x / r(x), x_{i}\right) \\
& =-\operatorname{Res}\left(x^{n} d x / r(x), \infty\right)= \begin{cases}0, & 0 \leq n<\operatorname{deg}(r)-1 \\
1, & n=\operatorname{deg}(r)-1 ;\end{cases}
\end{aligned}
$$

compare [Ser2, §III.6].

Invariant forms. Now suppose $x^{2}-4$ is not a square in $k$. Let $K=k(y)$ be the quadratic extension of $k$ obtained by adjoining a root of the equation

$$
y+\frac{1}{y}=x .
$$

Let $s(y) \in \mathbb{Z}[y]$ be the degree $2 d$ minimal polynomial for $y$ over $\mathbb{Q}$. Regarding $K \cong \mathbb{Q}[y] /(s(y))$ as a space of polynomials in $y$, let $F: K \rightarrow K$ be the multiplication map

$$
F(g(y))=y \cdot g(y) .
$$

Then $s(y)$ is the characteristic polynomial for $F$ as a linear endomorphism of $K / \mathbb{Q}$.

We will construct a lattice $B \subset K$ such that $F$ is an isometry of $B$. For the underlying group, we take

$$
B=\mathbb{Z}[y] /(s(y))=A \oplus A y \subset K .
$$

Then $F(B)=B$.

The Galois group of $K / k$ is generated by $\sigma(y)=1 / y$. Clearly $B^{\sigma}=B$, and the trace map

$$
\operatorname{Tr}_{k}^{K}(g)=g+g^{\sigma}=g(y)+g\left(y^{-1}\right)
$$

sends $B$ into $A$. We make $B$ into a lattice by defining the inner product:

$$
\left\langle g_{1}, g_{2}\right\rangle_{B}=\left\langle 1, \operatorname{Tr}_{k}^{K}\left(g_{1} g_{2}^{\sigma}\right)\right\rangle_{A}=\operatorname{Tr}_{\mathbb{Q}}^{K}\left(\frac{g_{1} g_{2}^{\sigma}}{r^{\prime}(x)}\right) .
$$


Then $F: B \rightarrow B$ is an isometry, because

$$
F\left(g_{1}\right) F\left(g_{2}\right)^{\sigma}=\left(y g_{1}(y)\right)\left(y g_{2}(y)\right)^{\sigma}=y g_{1}(y) y^{-1} g_{2}\left(y^{-1}\right)=g_{1} g_{2}^{\sigma} .
$$

Unimodularity. Our main concern is with automorphisms of unimodular lattices. Thus it is of interest to compute the discriminant of $B$.

Theorem 8.1 The lattice $B$ is even, with discriminant satisfying

$$
|\operatorname{disc}(B)|=\left|N_{\mathbb{Q}}^{k}\left(x^{2}-4\right)\right|=|r(2) r(-2)| .
$$

Here $N_{\mathbb{Q}}^{k}: A \rightarrow \mathbb{Z}$ is the norm map, defined by $N_{\mathbb{Q}}^{k}(f)=\prod_{1}^{d} f\left(x_{i}\right)$.

Proof. The inner product on $A$ makes $A^{2}$ into a unimodular lattice as well. Define $Q: A^{2} \rightarrow A^{2}$ by

$$
Q(a, b)=\left(\begin{array}{ll}
2 & x \\
x & 2
\end{array}\right)\left(\begin{array}{l}
a \\
b
\end{array}\right) .
$$

Then for $a+b y \in A \oplus A y=B$ we have

$$
(a+b y)(a+b y)^{\sigma}=a^{2}+b^{2}+a b x,
$$

and therefore

$$
\langle a+b y, a+b y\rangle_{B}=2\left\langle 1, a^{2}+b^{2}+a b x\right\rangle_{A}=\langle Q(a, b),(a, b)\rangle_{A^{2}} .
$$

Thus $B$ is even, and we have

$|\operatorname{disc}(B)|=|\operatorname{det}(Q)|=\left|N_{\mathbb{Q}}^{k}\left(4-x^{2}\right)\right|=\left|N_{\mathbb{Q}}^{k}(2-x) N_{\mathbb{Q}}^{k}(2+x)\right|=|r(2) r(-2)|$, since $N_{\mathbb{Q}}^{k}(n-x)=r(n)$.

Corollary 8.2 The following conditions are equivalent: (a) $B$ is an even, unimodular lattice; (b) $y+1$ and $y-1$ are units in $B$; (c) $x+2$ and $x-2$ are units in $A$; (d) $|r( \pm 2)|=1$; (e) $|s( \pm 1)|=1$.

Proof. Use the fact that $N_{\mathbb{Q}}^{K}(y \pm 1)=N_{\mathbb{Q}}^{k}(x \pm 2)$. 
Twisting by a unit. Let $u \in A^{\times}$be a unit in $A$. Then multiplication by $u$ is a symmetric automorphism of $A$ with determinant \pm 1 , so the lattice $A(u)$ with inner product

$$
\left\langle f_{1}, f_{2}\right\rangle_{A(u)}=\left\langle u f_{1}, f_{2}\right\rangle_{A}
$$

is still unimodular. Similarly, the lattice $B(u)$ with inner product

$$
\left\langle g_{1}, g_{2}\right\rangle_{B(u)}=\left\langle u g_{1}, g_{2}\right\rangle_{B}
$$

is still even, with $|\operatorname{disc}(B(u))|=|\operatorname{disc}(B)| ;$ and $F: B(u) \rightarrow B(u)$ is still an isometry, since $u^{\sigma}=u$.

Signature. In general the signature of $B(u)$ varies with the unit $u$. To calculate the signature, first observe that the lattice $B(u)$ determines a Hermitian inner product on $B(u) \otimes \mathbb{C}$. Using the fact that $F: B(u) \rightarrow B(u)$ is an isometry, we obtain an orthogonal, $F$-invariant decomposition

$$
B(u) \otimes \mathbb{C}=\bigoplus_{r(\tau)=0} E(\tau),
$$

where $E(\tau)=\operatorname{Ker}\left(F+F^{-1}-\tau I\right)$ is 2-dimensional, and the eigenvalues $\lambda, \lambda^{-1}$ of $F \mid E(\tau)$ satisfy $\lambda+\lambda^{-1}=\tau$.

Theorem 8.3 Let $\tau$ be a zero of $r(x)$. For $\tau \in \mathbb{R}$, the subspace $E(\tau) \subset$ $B(u) \otimes \mathbb{C}$ has signature

$(2,0) \quad$ if $|\tau|<2$ and $u(\tau) r^{\prime}(\tau)>0$;

$(0,2) \quad$ if $|\tau|<2$ and $u(\tau) r^{\prime}(\tau)<0$; and

$(1,1)$ otherwise.

For $\tau \notin \mathbb{R}$ the subspace $E(\tau) \oplus E(\bar{\tau})$ has signature $(2,2)$.

Proof. First suppose $\tau \in \mathbb{R}$. Then using the isomorphism $B=A+A y \cong A^{2}$ as in Theorem 8.1, we find the Hermitian inner product on $E(\tau) \cong \mathbb{C}^{2}$ comes from the complexification of the quadratic form

$$
q(a, b)=2 u(\tau)\left(a^{2}+b^{2}+a b \tau\right) / r^{\prime}(\tau)
$$

on $\mathbb{R}^{2}$. The signature of the form $\left(a^{2}+b^{2}+a b \tau\right)$ is $(1,1)$ if $|\tau|>2$ and $(2,0)$ if $|\tau|<2$. The signature of $q(a, b)$ is the same, unless $u(\tau) / r^{\prime}(\tau)<0$, in which case it is reversed. 
Now suppose $\tau \notin \mathbb{R}$, and let $S \subset E(\tau) \oplus E(\bar{\tau})$ be the span of the $(\lambda, \bar{\lambda})$ eigenvectors for $F$, where $\lambda+\lambda^{-1}=\tau$. Since $\tau \notin \mathbb{R}, \bar{\lambda}$ is distinct from both $\lambda$ and $\lambda^{-1}$; therefore $S$ is a 2-dimensional isotropic subspace, and thus $E(\tau) \oplus E(\bar{\tau})$ has signature $(2,2)$.

Corollary 8.4 The lattice $B(u)$ has signature $(d, d)+(p,-p)+(-q, q)$, where $p$ is the number of roots of $r(x)$ in $[-2,2]$ satisfying $u(\tau) r^{\prime}(\tau)>0$, and $q$ is the number satisfying $u(\tau) r^{\prime}(\tau)<0$.

Unramified Salem polynomials. As an application, we show certain Salem numbers can be realized as eigenvalues of automorphisms of unimodular lattices. Following Corollary 8.2, we say a Salem polynomial $S(y)$ (or the corresponding Salem number) is unramified if $|S( \pm 1)|=1$; and a Salem trace polynomial $R(x)$ is unramified if $|R( \pm 2)|=1$.

Theorem 8.5 Let $S(y)$ be an unramified Salem polynomial of degree $2 d$. Then $d$ is odd, and $S(y)$ is the characteristic polynomial of an automorphism of the even, unimodular lattice $L$ of signature $(d, d)$.

Proof. Let $R(x)$ be the associated Salem trace polynomial of degree $d$, with roots $\tau_{1}, \ldots, \tau_{d-1}$ in $(-2,2)$ and $\tau_{d}>2$. Since $R(x)$ has no roots in the interval $(-\infty,-2]$ and exactly one root in $(2, \infty)$, we have $R(2)<0$ and $R(-2)(-1)^{d}>0$. Since $S(y)$ is unramified, $|R( \pm 2)|=1$, and thus $R(-2)=(-1)^{d}, R(2)=-1$. But $R(2)-R(-2)=0 \bmod 4$ and therefore $d$ is odd.

Now let $B=\mathbb{Z}[y] / S(y)$ and $F(g)=y g$ as above. Then $F$ is an isometry of the even, unimodular lattice $L=B(1)$. Since $R^{\prime}(x)$ alternates sign at the zeros of $R(x)$, the signatures of the summands $E\left(\tau_{i}\right)$ of $B(1) \otimes \mathbb{C}$ alternate between $(2,0)$ and $(0,2)$ for $i=1, \ldots, d-1$. The last summand $E\left(\tau_{d}\right)$ has signature $(1,1)$, so altogether $B(1)$ has signature $(d, d)$.

Ramification and parity. Here is another explanation for the parity constraint on unramified Salem polynomials. If $S(y)$ has degree $2 d$ and $d$ is even, then $R(x)$ has an odd number of zeros in the range $[-2,2]$, and therefore the lattice $B(1)$ has signature $(d-1, d+1)$. But an even unimodular lattice of signature $(p, q)$ satisfies $p+q=0 \bmod 8$, so $S(y)$ must be ramified.

Sextic Salem numbers, reprise. The simplest unramified Salem polynomials are the sextics of the form

$$
S(y)=y^{6}-a y^{5}-y^{4}+(2 a-1) y^{3}-y^{2}-a y+1, \quad a \geq 0 .
$$


There are no unramified Salem polynomials of lower degree, and these are the only unramified Salem polynomials of degree 6 .

In $\S 4$ we showed $S(y)$ arises as the characteristic polynomial of $F^{*} \mid H^{2}(Y, \mathbb{Z})$, where $F: Y \rightarrow Y$ is an automorphism of a complex torus. Noting that the intersection pairing makes $H^{2}(Y, \mathbb{Z})$ into an even, unimodular lattice of signature $(3,3)$, we obtain a geometric instance of the result above.

\section{From Salem numbers to automorphisms}

Consolidating the preceding results, we can now give

- a general construction of K3 surface automorphisms from unramified Salem numbers, and

- a criterion for the resulting automorphism to have a Siegel disk.

Theorem 9.1 Let $R(x), U(x) \in \mathbb{Z}[x]$ be a pair such that:

- $R(x)$ is an unramified degree 11 Salem trace polynomial;

- $U(x)$ represents a unit in $\mathbb{Z}[x] /(R(x))$; and

- there is a unique root $\tau$ of $R(x)$ in $[-2,2]$ such that $U(\tau) R^{\prime}(\tau)>0$.

Then there exists a K3 surface automorphism $f: X \rightarrow X$ such that

- $\delta(f)+\delta(f)^{-1}=\tau$, and

- $S(x)=\operatorname{det}\left(x I-f^{*} \mid H^{2}(X)\right)$,

where $S(x)$ is the degree 22 Salem polynomial associated to $R(x)$.

Note that $S(x)=x^{11} R\left(x+x^{-1}\right)$.

Proof. The trace-form construction of $\S 8$ yields a lattice automorphism $F: B(U) \rightarrow B(U)$ with characteristic polynomial $S(x)$. Since $R(x)$ is unramified, $B(U)$ is an even, unimodular lattice, with signature $(3,19)$ by Corollary 8.4.

Using the Torelli theorem, surjectivity of the period mapping, and uniqueness of the even unimodular $(3,19)$ lattice, Theorem 3.4 (Synthesis) yields a K3 surface automorphism $f: X \rightarrow X$ and a marking $\iota: H^{2}(X, \mathbb{Z}) \rightarrow B(U)$ such that $F=\iota \circ f^{*} \circ \iota^{-1}$. Thus $S(x)$ is also the characteristic polynomial of $f^{*} \mid H^{2}(X)$. 
By Theorem 8.3, the eigenspaces of $F+F^{-1}$ are 2-dimensional, and

$$
E(\tau)=\operatorname{Ker}\left(F+F^{-1}-\tau I\right) \subset B(U) \otimes \mathbb{C}
$$

is the unique eigenspace of $F+F^{-1}$ with signature $(2,0)$. Similarly, $H^{2,0}(X) \oplus$ $H^{0,2}(X) \subset H^{2}(X)$ is the unique eigenspace of $f^{*}+\left(f^{*}\right)^{-1}$ with signature $(2,0)$. Thus $\iota^{-1}(E(\tau))=H^{2,0}(X) \oplus H^{0,2}(X)$ and therefore $\delta(f)+\delta(f)^{-1}=\tau$.

Traces. The trace of a monic polynomial $P(x)=x^{d}+a_{1} x^{d-1}+\cdots+a_{d}$ is $-a_{1}$, the sum of its roots. The traces of $R(x)$ and $S(x)$ agree and coincide with $\operatorname{Tr}\left(f^{*} \mid H^{2}(X)\right)$. Continuing from the previous result, the cohomological criterion for a Siegel disk given by Theorem 7.1 implies:

Theorem 9.2 Suppose in addition that $R(x)$ and $\tau$ satisfy:

- The trace of $R(x)$ is -1 ,

- $\tau>1-2 \sqrt{2}=-1.8284271 \ldots$, and

- $R(x)$ has a root $\tau^{\prime}<1-2 \sqrt{2}$.

Then $f$ has a unique fixed-point $p \in X$, and $p$ is the center of a Siegel disk.

\section{Examples of Siegel disks}

In this section we exhibit polynomials $R(x)$ and $U(x)$ satisfying Theorem 9.2 and thereby prove:

Theorem 10.1 There exist K3 surface automorphisms with Siegel disks.

Table of Salem polynomials and units. Table 4 presents 10 examples of K3 surface automorphisms with Siegel disks. Each example occupies three rows.

The first two rows give the coefficients of a degree 22 Salem polynomial $S(x)$ and the associated degree 11 Salem trace polynomial $R(x)$. Since the coefficients of $S(x)$ are symmetric - that is, the coefficients of $x^{i}$ and $x^{22-i}$ agree - we only give them up to $x^{11}$.

The third row exhibits a unit $U(x)$ compatible with $R(x)$, meaning there is a unique root $\tau$ of $R(x)$ in $[-2,2]$ such that $R^{\prime}(\tau) U(\tau)>0$. (In general there are many such units.) 
For each example, the pair $(R(x), U(x))$ satisfies the hypotheses of Theorem 9.2 , and so it determines a K3 surface automorphism $f: X \rightarrow X$ with a Siegel disk. The Salem polynomial $S(x)$ is the characteristic polynomial of $f \mid H^{2}(X)$; its largest root $\lambda(f)$ is a Salem number, given in the first column of the table. The topological entropy $\log \lambda(f)$ is a natural measure of the complexity of $f$, so we have ordered the table with $\lambda(f)$ increasing.

Detailed example. To explain the table more fully, we present some details regarding the degree 22 Salem number $\lambda \approx 1.37289$, the first example listed. This Salem number is a root of the polynomial

$$
\begin{aligned}
S(x)= & 1+x-x^{3}-2 x^{4}-3 x^{5}-3 x^{6}-2 x^{7}+2 x^{9}+4 x^{10}+5 x^{11} \\
& +4 x^{12}+2 x^{13}-2 x^{15}-3 x^{16}-3 x^{17}-2 x^{18}-x^{19}+x^{21}+x^{22} .
\end{aligned}
$$

The corresponding Salem trace polynomial, satisfied by $\lambda+\lambda^{-1}$, is

$$
\begin{aligned}
R(x)= & -1-8 x+24 x^{2}+42 x^{3}-54 x^{4}-66 x^{5}+40 x^{6}+42 x^{7}-11 x^{8} \\
& -11 x^{9}+x^{10}+x^{11} .
\end{aligned}
$$

Note that both polynomials have trace -1 and are unramified - for example, $R( \pm 2)=-1$. The graph of $R(x)$, displaying its 11 real roots, is shown in Figure 5. All roots except $\lambda+\lambda^{-1} \approx 2.10128$ lie in $[-2,2]$, as required.

A unit compatible with $R(x)$ is given by

$$
U(x)=-2 x+6 x^{3}-5 x^{5}+x^{7} .
$$

To verify that $U(x) \in \mathbb{Z}[x] /(R(x))$ is a unit, one can check that $\left|\operatorname{det} U\left(M_{R}\right)\right|=$ 1 , where $M_{R}$ is the $11 \times 11$ companion matrix of $R$.

There is a unique root $\tau \approx-1.66716$ of $R(x)$ in $[-2,2]$ with $R^{\prime}(\tau) U(\tau)>$ 0 . On the other hand, there are two roots of $R(x)$ smaller than $1-2 \sqrt{2} \approx$ -1.82843 , the smallest being $\tau^{\prime} \approx-1.97098$.

Thus the hypotheses of Theorem 9.2 are satisfied, and we obtain an example of a K3 surface automorphism $f: X \rightarrow X$ with a Siegel disk. In fact we obtain a pair of complex-conjugate examples, one for each root of the equation $\delta(f)+\delta(f)^{-1}=\tau$. For the $\operatorname{root} \delta(f) \approx \exp (2.55635 i)$, we obtain a Siegel disk centered at $p \in X$ with the eigenvalues of $D f_{p}$ given by

$$
\{\alpha, \beta\} \approx\{\exp (-2.81769 i), \exp (-0.909141 i)\},
$$

according to equation (7.1).

Finiteness. Note that the set of Salem numbers of degree 22 and trace -1 is finite. More generally, the set of Salem polynomials $S(x)$ of degree $d$ and 


\begin{tabular}{|c|c|c|c|c|c|c|c|c|c|c|c|c|}
\hline$\lambda$ & $x^{0}$ & $x^{1}$ & $x^{2}$ & $x^{3}$ & $x^{4}$ & $x^{5}$ & $x^{6}$ & $x^{7}$ & $x^{8}$ & $x^{9}$ & $x^{10}$ & $x^{11}$ \\
\hline \multirow[t]{3}{*}{1.37289} & 1 & 1 & 0 & -1 & -2 & -3 & -3 & -2 & 0 & 2 & 4 & 5 \\
\hline & -1 & -8 & 24 & 42 & -54 & -66 & 40 & 42 & -11 & -11 & 1 & 1 \\
\hline & 0 & -2 & 0 & 6 & 0 & -5 & 0 & 1 & & & & \\
\hline \multirow[t]{3}{*}{1.45099} & 1 & 1 & -1 & -3 & -3 & -1 & 2 & 4 & 4 & 1 & -3 & -5 \\
\hline & -5 & -4 & 49 & 37 & -100 & -81 & 58 & 50 & -13 & -12 & 1 & 1 \\
\hline & 2 & 0 & -9 & 14 & 23 & -23 & -25 & 9 & 9 & -1 & -1 & \\
\hline \multirow[t]{3}{*}{1.48115} & 1 & 1 & 0 & -2 & -3 & -3 & -2 & -1 & 0 & 2 & 4 & 5 \\
\hline & -1 & 4 & 36 & 23 & -73 & -58 & 48 & 41 & -12 & -11 & 1 & 1 \\
\hline & 0 & -1 & 3 & 6 & -4 & -5 & 1 & 1 & & & & \\
\hline \multirow[t]{3}{*}{1.52612} & 1 & 1 & 0 & -2 & -4 & -4 & -2 & 1 & 3 & 3 & 2 & 1 \\
\hline & -1 & 0 & 20 & 12 & -65 & -51 & 47 & 40 & -12 & -11 & 1 & 1 \\
\hline & 0 & -1 & -5 & 17 & 37 & -12 & -33 & 2 & 10 & 0 & -1 & \\
\hline \multirow[t]{3}{*}{1.55377} & 1 & 1 & -1 & -3 & -3 & -1 & 1 & 1 & 1 & 1 & 1 & 1 \\
\hline & -5 & 4 & 61 & 39 & -103 & -82 & 58 & 50 & -13 & -12 & 1 & 1 \\
\hline & 3 & 3 & -6 & 16 & 37 & -12 & -33 & 2 & 10 & 0 & -1 & \\
\hline \multirow[t]{3}{*}{1.60709} & 1 & 1 & 0 & -2 & -4 & -5 & -4 & -1 & 2 & 4 & 5 & 5 \\
\hline & -1 & -4 & 20 & 21 & -61 & -53 & 46 & 40 & -12 & -11 & 1 & 1 \\
\hline & 0 & -7 & 8 & 34 & -6 & -32 & 1 & 10 & 0 & -1 & & \\
\hline \multirow[t]{3}{*}{1.6298} & 1 & 1 & -1 & -3 & -3 & -2 & -1 & 0 & 2 & 3 & 2 & 1 \\
\hline & -9 & -8 & 58 & 50 & -98 & -84 & 57 & 50 & -13 & -12 & 1 & 1 \\
\hline & -4 & -6 & 16 & 14 & -20 & -7 & 8 & 1 & -1 & & & \\
\hline \multirow[t]{3}{*}{1.6458} & 1 & 1 & -1 & -4 & -5 & -2 & 4 & 8 & 7 & 1 & -6 & -9 \\
\hline & -1 & 8 & 40 & 2 & -110 & -65 & 65 & 48 & -14 & -12 & 1 & 1 \\
\hline & -2 & 2 & 21 & -4 & -25 & 1 & 9 & 0 & -1 & & & \\
\hline \multirow[t]{3}{*}{1.66566} & 1 & 1 & -2 & -5 & -3 & 3 & 7 & 4 & -2 & -4 & -1 & 1 \\
\hline & -1 & 32 & 112 & 36 & -164 & -103 & 78 & 59 & -15 & -13 & 1 & 1 \\
\hline & -8 & -33 & -23 & 49 & 62 & -22 & -42 & 3 & 11 & 0 & -1 & \\
\hline \multirow[t]{3}{*}{1.69496} & 1 & 1 & -1 & -4 & -5 & -2 & 3 & 6 & 5 & 1 & -3 & -5 \\
\hline & -1 & 12 & 48 & 5 & -112 & -66 & 65 & 48 & -14 & -12 & 1 & 1 \\
\hline & 0 & 1 & -18 & -7 & 48 & 14 & -35 & -7 & 10 & 1 & -1 & \\
\hline
\end{tabular}

Table 4. Salem polynomials and units: $(S(x), R(x), U(x))$. 


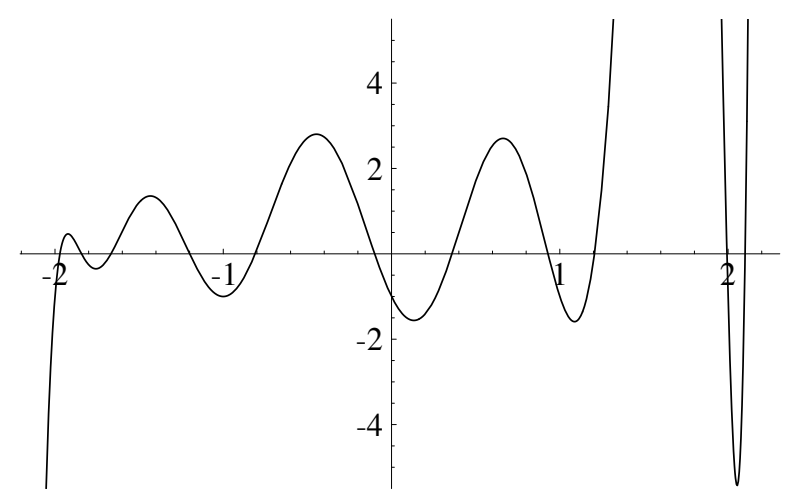

Figure 5. Graph of the degree 11 Salem trace polynomial $R(x)$.

trace $t$ is finite, since the trace controls the size of the unique root outside the unit circle, and the size of the roots controls the size of the (integral) coefficients of $S(x)$.

One can also show that $S(x)=\operatorname{det}\left(x I-f^{*} \mid H^{2}(X)\right)$ determines the automorphism $f: X \rightarrow X$ up to a finite number of choices. Thus Theorem 9.2 yields only a finite number of Siegel disk examples up to isomorphism.

Searching for Salem numbers. Table 4 is the result of an intensive computer search for unramified degree 22 Salem numbers of trace -1 . Altogether we located 51 such numbers, ranging from 1.37289 to 2.51503 ; the first 10 appear in the table. Sturm's method, based on alternations of signs [La, XI.2], was used to count the number of roots of a candidate Salem trace polynomial $R(x)$ in the interval $[-2,2]$.

All 51 Salem numbers we found arise from K3 surface automorphisms with Siegel disks. That is, for each of the corresponding Salem trace polynomials $R(x)$, there was a compatible unit $U(x)$ satisfying Theorem 9.2.

Generally many units are compatible with a given $R(x)$, allowing one to vary $\tau=\delta(f)+\delta(f)^{-1}$ through different roots of $R(x)$ and to obtain several distinct K3 surface automorphisms for the same Salem polynomial. On the other hand, units which differ by a square give isomorphic lattice automorphisms $F: B(U) \rightarrow B(U)$, so only the class of $U$ in the finite group $A^{\times} /\left(A^{\times}\right)^{2}$ is relevant.

To find $U(x)$, we used an algebra package to obtain a basis for the unit group of the order $A=\mathbb{Z}[x] /(R(x))$ in the totally real field $k=\mathbb{Q}[x] /(R(x))$. It was then straightforward to search the cosets of $A^{\times} /\left(A^{\times}\right)^{2}$ for a compatible unit. 
Negative trace. We remark that Salem numbers with negative trace seem to be rather rare. Among the 630 degree 22 Salem polynomials with coefficients satisfying $\left|a_{i}\right| \leq 1,596$ have trace 1, 33 have trace 0 and only one has trace -1 , namely

$$
\begin{aligned}
S(x)= & 1+x-x^{3}-x^{4}-x^{5}-x^{6}-x^{7}-x^{8}-x^{9}-x^{10}-x^{11} \\
& -x^{12}-x^{13}-x^{14}-x^{15}-x^{16}-x^{17}-x^{18}-x^{19}+x^{21}+x^{22} .
\end{aligned}
$$

On the other hand, Smyth has shown there exist infinitely many Salem numbers with trace -1 [Smy].

Question. Are there any Salem numbers with trace less than -1 ?

\section{Limits of Kähler-Einstein metrics}

In this section we present an application of dynamics to the differential geometry of K3 surfaces.

Kähler-Einstein metrics. Let $g$ be a Kähler metric on a compact complex manifold $X$, with associated symplectic $(1,1)$-form $\omega_{g}$. Then $g$ is a KählerEinstein metric if there is a constant $\kappa \in \mathbb{R}$ such that

$$
\operatorname{Ric}(g)=\kappa \cdot \omega_{g},
$$

where $\operatorname{Ric}(g)=-\partial \bar{\partial} \log \operatorname{det} g$ is the Ricci form of $g$.

Now suppose $X$ is a K3 surface. Then by triviality of the canonical bundle, $g$ is a Kähler-Einstein metric $\Longleftrightarrow \operatorname{Ric}(g)=0 \Longleftrightarrow$ the volume form $\omega_{g}^{2}$ of $g$ is proportional to $\eta \wedge \bar{\eta}$, where $\eta \neq 0$ is a holomorphic $(2,0)$ form on $X$. Such metrics exist in abundance, according to:

Theorem 11.1 (Yau) Every cohomology class in the Kähler cone $C_{X} \subset$ $H^{1,1}(X)$ is represented by the $(1,1)$-form $\omega$ of a Kähler-Einstein metric.

See $[\mathrm{Yau}],[\mathrm{Bo}]$.

It would be interesting to understand the behavior of a Kähler-Einstein metric as its cohomology class $[\omega]$ tends to the boundary of Kähler cone. Here we will show that the metric can collapse to zero on an open set, if $X$ comes from one of the Siegel disk examples of $\S 10$.

Theorem 11.2 Let $f: X \rightarrow X$ be a K3 surface automorphism with a Siegel disk $U$. Then there exists a sequence of Kähler-Einstein metrics on $X$, with symplectic forms satisfying

$$
\left[\omega_{n}\right] \rightarrow \xi \in \partial C_{X} \subset H^{1,1}(X), \quad \xi \neq 0,
$$

such that $\omega_{n} \mid U \rightarrow 0$ uniformly on compact sets. 
Proof. Since $f$ has a Siegel disk, we have $\lambda=\lambda(f)>1$ (Theorem 7.3). Consider any Kähler-Einstein metric $g_{0}$ on $X$ with $(1,1)$-form $\omega_{0}$, and let $\omega_{n}=\lambda^{-n}\left(f^{n}\right)^{*}\left(\omega_{0}\right)$. Then $\omega_{n}$ is also the $(1,1)$-form of a Kähler-Einstein metric $g_{n}$, since the condition $\operatorname{Ric}\left(g_{0}\right)=0$ is preserved under pulling back.

We claim the cohomology classes $\left[\omega_{n}\right]$ converge to an eigenvector $\xi \neq 0$ in $\partial C_{X}$ satisfying $f^{*}(\xi)=\lambda \xi$. Indeed, $f^{*}$ preserves the intersection form of signature $(1,19)$ on $H^{1,1}(X)$, and therefore it determines an isometry $F$ of the hyperbolic space

$$
\mathbb{H}^{19} \subset \mathbb{P} H^{1,1}(X)_{\mathbb{R}}
$$

Since we have $\lambda>1, F$ translates along a geodesic $\gamma \subset \mathbb{H}^{19}$ joining points $p_{ \pm} \in \partial \mathbb{H}^{19}$, corresponding to the $\lambda^{ \pm 1}$-eigenspaces of $f$. For any point $q \in$ $\mathbb{H}^{19}$, including that represented by $\left[\omega_{0}\right]$, we have $F^{n}(q) \rightarrow p_{+}$. Since $\left[\omega_{n}\right]$ is $\left[\left(f^{n}\right)^{*}\left(\omega_{0}\right)\right]$ renormalized by the leading eigenvalue of $f^{*}$, it converges to a $\lambda$-eigenvector $\xi$.

Since $f \mid U$ is conjugate to a rotation, any compact set $K^{\prime} \subset U$ can be enlarged to an $f$-invariant compact set $K \subset U$. Moreover there is a smooth $f$-invariant metric on $U$. Using this invariant metric to measure the size of $\omega_{n}$, we have:

$$
\left\|\omega_{n}\left|K\left\|_{\infty}=\lambda^{-n}\right\| \omega_{0}\right| K\right\|_{\infty} \rightarrow 0
$$

as claimed. (In fact $\omega_{n} \mid K \rightarrow 0$ in the $C^{\infty}$ topology.)

Eigencurrents. By a result of Cantat, the sequence $\omega_{n}=\lambda^{-n}\left(f^{n}\right)^{*}\left(\omega_{0}\right)$ just constructed actually converges to a natural current $\omega_{\infty}=C_{+} \alpha_{+}$representing the cohomology class $\xi$.

Theorem 11.3 (Cantat) Let $f: X \rightarrow X$ be a K3 surface automorphism with $\lambda=\lambda(f)>1$. Then:

- There exists a pair of closed, positive $(1,1)$-currents $\alpha_{-}, \alpha_{+}$, unique up to scale, such that $f^{*}\left(\alpha_{ \pm}\right)=\lambda^{ \pm 1} \alpha_{ \pm}$.

- For any other closed, positive $(1,1)$ current $\omega$, there are constants $C_{ \pm}$ such that $\lambda^{-|n|}\left(f^{ \pm n}\right)^{*}(\omega) \rightarrow C_{ \pm} \alpha_{ \pm}$as $n \rightarrow \infty$.

(See [Ca1, §4.2-4.4], or observe that [Ca2, Theorem 2], while stated for projective surfaces, also applies to Kähler surfaces.)

So we also have:

Theorem 11.4 Let $f: X \rightarrow X$ be a K3 surface automorphism with a Siegel disk $U$. Then the eigencurrents $\alpha_{+}$and $\alpha_{-}$vanish on $U$. 


\section{References}

[AB] M. F. Atiyah and R. Bott. A Lefschetz fixed point formula for elliptic complexes II. Ann. of Math. 88(1968), 451-491.

[BPV] W. Barth, C. Peters, and A. van de Ven. Compact Complex Surfaces. Springer-Verlag, 1984.

[Bv1] Beauville. Some remarks on Kähler manifolds with $c_{1}=0$. In Classification of Algebraic and Analytic Manifolds (Katata, 1982), pages 1-26. Birkhäuser, 1983.

[Bv2] A. Beauville et al. Géométrie des surfaces K3: modules et périodes, volume 126. Astérisque, 1985.

[Bo] J. Bourguingon et al. Première classe de Chern et courbure de Ricci: preuve de la conjecture de Calabi. Astérisque, volume 58, Société Mathématique de France, 1978.

[Br] R. J. Brown. Anosov mapping class actions on the $S U(2)$ representation variety of a punctured torus. Ergod. Th. \& Dynam. Sys. 18(1998), 539-554.

[Ca1] S. Cantat. Dynamique des automorphismes des surfaces complexes compactes. Thesis, ENS Lyon, 1999.

[Ca2] S. Cantat. Dynamique des automorphismes des surfaces projectives complexes. CRAS Paris Sér. I Math. 328(1999), 901-906.

[Fe] H. I. Fel'dman. An improvement of the estimate of a linear form in the logarithms of algebraic numbers. Math. USSR Sb. 6(1968), 393-406.

[Fr] D. Fried. Word maps, isotopy and entropy. Trans. Amer. Math. Soc. 296(1986), 851-859.

[Frl] S. Friedland. Entropy of algebraic maps. In Proceedings of the Conference in Honor of Jean-Pierre Kahane (Orsay, 1993), pages 215-228. J. Fourier Anal. Appl., 1995.

[Gr] M. Gromov. On the entropy of holomorphic maps. L'Enseignement Mathématique 49(2003), 217-235. 
[GM] B. Gross and C. McMullen. Automorphisms of even unimodular lattices and unramified Salem numbers. J. Algebra 257(2002), 265290 .

[Her] M. Herman. Recent results and some open questions on Siegel's linearization theorem of germs of complex analytic diffeomorphisms of $\mathbf{C}^{n}$ near a fixed point. In VIIIth International Congress on Mathematical Physics (Marseille, 1986), pages 138-184. World Sci. Publishing, 1987.

[La] S. Lang. Algebra. Addison-Wesley, 1984.

[Mak1] R. S. MacKay. Hyperbolic structure in classical chaos. In Quantum Chaos (Varenna, 1991), pages 1-50. North-Holland, 1993.

[Mak2] R. S. MacKay. Renormalisation in Area-Preserving Maps. World Scientific, 1993.

[Man] R. Mañé. Ergodic Theory and Differentiable Dynamics. SpringerVerlag, 1987.

[Maz] B. Mazur. The topology of rational points. J. Exp. Math. 1(1992), $35-46$.

[Mc] C. McMullen. Complex Dynamics and Renormalization, volume 135 of Annals of Math. Studies. Princeton University Press, 1994.

[Mil] J. Milnor. Dynamics in One Complex Variable: Introductory Lectures. Vieweg, 1999.

[MH] J. Milnor and D. Husemoller. Symmetric Bilinear Forms. Springer, 1973.

[Sa] R. Salem. Algebraic Numbers and Fourier Analysis. Wadsworth, 1983.

[Ser1] J. P. Serre. A Course in Arithmetic. Springer-Verlag, 1973.

[Ser2] J. P. Serre. Local Fields. Springer-Verlag, 1979.

[Sil] J. Silverman. Rational points on K3 surfaces: A new canonical height. Invent. math. 105(1991), 347-373.

[Smy] C. J. Smyth. Salem numbers of negative trace. Math. Comp. 69(2000), 827-838. 
[St] S. Sternberg. Infinite Lie groups and the formal aspects of dynamical systems. J. Math. Mech. 10(1961), 451-474.

[Ti] R. Tijdeman. On the Gel'fond-Baker method and its applications. In F. Browder, editor, Mathematical Developments Arising from Hilbert Problems, volume 28 of Proc. Symp. Pure Math. Amer. Math. Soc., 1976.

[Wa] L. Wang. Rational points and canonical heights on K3-surfaces in $\mathbb{P}^{1} \times \mathbb{P}^{1} \times \mathbb{P}^{1}$. In Recent Developments in the Inverse Galois Problem, pages 273-289. Amer. Math. Soc., 1995.

[Yau] S.-T. Yau. On the Ricci-curvature of a complex Kähler manifold and the complex Monge-Ampère equation. Comment. Pure Appl. Math. 31(1978), 339-411.

[Ym] Y. Yomdin. Volume growth and entropy. Israel J. Math. 57(1987), 285-300.

Mathematics Department

HARVARD UNIVERSITY

1 OXFORD ST

CAmbridge, MA 02138-2901 